\title{
Application of Neutron Correlation Techniques to Warhead Authentication: Feasibility and Conceptual Requirements-Monte Carlo Simulations and Statistical Analysis
}

M. I. Frank and J. K. Wolford, Jr,

U.S. Department of Energy August 13, 2004

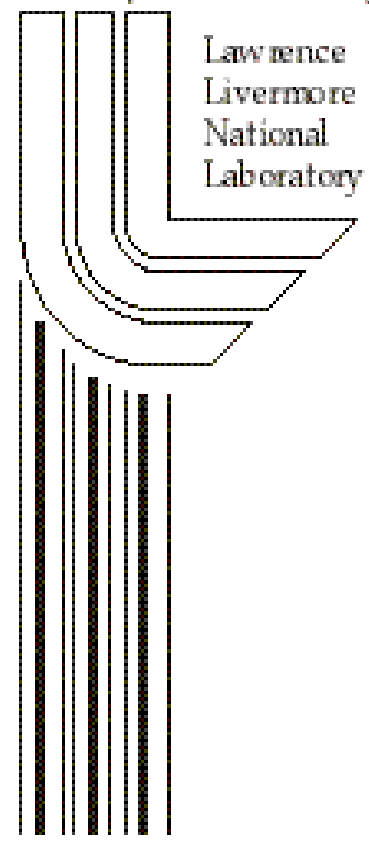




\section{DISCLAIMER}

This document was prepared as an account of work sponsored by an agency of the United States Government. Neither the United States Government nor the University of California nor any of their employees, makes any warranty, express or implied, or assumes any legal liability or responsibility for the accuracy, completeness, or usefulness of any information, apparatus, product, or process disclosed, or represents that its use would not infringe privately owned rights. Reference herein to any specific commercial product, process, or service by trade name, trademark, manufacturer, or otherwise, does not necessarily constitute or imply its endorsement, recommendation, or favoring by the United States Government or the University of California. The views and opinions of authors expressed herein do not necessarily state or reflect those of the United States Government or the University of California, and shall not be used for advertising or product endorsement purposes.

This work was performed under the auspices of the U. S. Department of Energy by the University of California, Lawrence Livermore National Laboratory under Contract No. W-7405-Eng-48. 


\title{
Application of Neutron Correlation Techniques to Warhead Authentication: Feasibility and Conceptual Requirements-Monte Carlo Simulations and Statistical Analysis
}

\author{
Michael I. Frank ${ }^{\ddagger}$ and James K. Wolford, Jr. ${ }^{\dagger}$ \\ Lawrence Livermore National Laboratory, P.O. Box 808, Livermore, CA 94551
}

\begin{abstract}
We explore the feasibility of using Feynman moments as attributes of fissile material in warhead authentication measurements. We present results of computer simulations of neutron correlation measurements to validate and inform the application of the method to measurements in an arms control scenario. We establish the robustness of the method for use in automated measuring equipment that protects classified or sensitive data using an information barrier. Drawing from our results, we define high-level requirements to govern the design process, and guide the construction of a prototype.
\end{abstract}

Keywords: neutron correlation, fissile material detection, Feynman technique

\section{BACKGROUND AND OBJECTIVE}

Warhead authentication, like all verification or transparency activities, involves prescribed measurements. Typically the measurements depend on properties of nuclear material or warheads sealed in specially designed containers that promote safety and conceal the specific shape and extent of the contents. (Concealing a classified or sensitive item serves to protect any sight sensitive aspects.) Identifying the measurement techniques to be used often requires detailed negotiations between the parties to an agreement. A chief concern is the protection of sensitive information implicit in the shape and composition of the measured object. When a controlled item is presented for inspection, it will typically be sealed in a container. Without opening this container, the inspecting party must determine if the item matches the declaration, and must do so without gleaning information deemed sensitive by the declaring party. Sensitive information typically includes the isotopic composition of nuclear materials in strategic weapons, their age, and configuration. Unfortunately, these are some of the very attributes that an inspection would normally seek to determine, since they characterize weapon grade material in a classified form.

A distinguishing characteristic of nuclear weapons is the presence of fissile material. In the presence of a neutron source and in certain configurations, such material will give rise to "chains" of neutron multiplication. While this indication alone does not prove that an item in a container is a warhead, a correlation analysis of neutron events using the Feynman Moment technique will provide higher confidence in the composition and amount of material present than the less involved neutron methods that have been applied to date. Nevertheless, this technique alone will not provide all the information one might need for a positive determination. For example, it will not by itself disclose the presence of high explosive. Therefore in proposing this type of measurement for warhead authentication, we assume that it would be one of several inspection techniques applied.

Typical radiation-based methods for verifying the presence of specific nuclear materials include gross counting of neutrons and gamma rays, gamma ray spectroscopy (and to a lesser extent neutron spectroscopy with some form of template analysis), and time-resolved measurements of neutrons and gamma rays. The nuclear material in each case may be a passive emitter, or, particularly for uranium, the signature producing reactions may be induced using a neutron or high-energy gamma source, suitably time gated. Other physical methods are available to characterize the weight, mass, and configuration of nuclear weapons components, including radiographic imaging, weighing, measurement of moments of inertia, thermal measurements, and electromagnetic measurements.

The technique of neutron correlation counting is notable and distinct from these other methods because it is a direct measure of the capability for sustaining induced fission multiplication. This is an essential characteristic of material from nuclear weapons. Moreover, correlation counting does not require detailed knowledge of the isotopic composition of the

\footnotetext{
†e-mail: mfrank@1lnl.gov; telephone: (925) 424-5853; fax: (925) 423-2759
}

†e-mail: wolford@llnl.gov; telephone: (925) 422-7236; fax: (925) 423-2759 
materials being assessed. This is significant for authentication measurements where access to the measured items is strictly limited.

Data from neutron measurements of nuclear warheads contains classified information, just like any other detailed physical measurement. We expect that an agreement to practice warhead authentication would necessarily include strong measures to protect that information. To allow sufficiently detailed measurements within these protections, an information barrier may be employed. An information barrier is formally defined as "technology and procedures that prevent the release of host country classified information to a monitoring party during a joint inspection of a sensitive item, while promoting assurance of an accurate assessment of host country declarations regarding the item." ${ }^{\dagger}$ Practically speaking, an information barrier conceals all but a few simple and unclassified measurement indications from the outside world, i.e., equipment operators and witnesses. Typically, the results of data analysis are compared to threshold values to determine whether the measured item matches the declaration. The instrument display consists of one or more binary indicators giving the result of the threshold test. This means that the process of gathering and analyzing data is hidden, and the stakeholders must believe in its integrity. This assurance comes through robust measurement techniques, sound instrument design, and defined procedures for periodically demonstrating that the entire measurement system, including the concealed portions, is operating according to agreed specification.

In general, we have determined that neutron correlation analysis using a generalized form of the Feynman Moments technique developed at LLNL can provide an effective, robust, technique for warhead authentication that compliments traditional gamma-ray spectroscopy, and may be a feasible stand-alone method for certain applications. Furthermore, we have shown that the technique can be made sufficiently automatic to use in a measurement system equipped with an information barrier. Our reservations are that the correlation method can depend strongly on material configuration, and that identical masses of fissionable material may yield different results if they are configured in different geometries.

\section{Objective and Summary Assessment}

The objective of this study is to provide an initial assessment of the effectiveness and feasibility of neutron correlation techniques for warhead authentication. Based on our results, we also provide high-level requirements for a warhead authentication system and propose follow-on detector analysis and development. In summary, we have answered four key questions with regard to neutron correlation methods for warhead authentication, with the following results:

1. "Can neutron correlation analysis be used for detection of fissionable, multiplying nuclear material in a container?"

Yes. We have shown that the technique should succeed for the distances, quantities of material, and amounts of shielding likely to be present in a Warhead Authentication measurement scenario.

2. "If feasible, is the method accurate enough, in expected situations, to provide the levels of assurance needed for arms control? In particular, can it distinguish a warhead from a spoof"

Yes. However, the neutron correlation method alone may not be sufficient to determine all conceivable attributes of an object that one might wish to use in a Warhead Authentication application. Thus far, we have limited our analysis to a set of canonical detection scenarios and focused on the quantity and quality of fissile material present. In all cases we showed a robust, high-confidence capability for rejection of non-warhead "spoof" items. With further work, we may find the technique useful for characterizing additional, unique properties of warheads, but we assume here that neutron correlation would be one of several measurements performed.

3. "If assurance is adequate, can the method be automated to the point where data collection and processing can proceed in the presence an information barrier?"

Yes. The key requirements for the inclusion of an information barrier in the design of a measurement instrument are twofold: 1. The system must support data collection, processing, and extraction of attributes or a template without operator intervention, and with a minimum of controls and indications. 2. The system must allow for selfauthentication using known reference items. Our simulation of the measurement process shows that both of these goals can be met, in principle. The baseline requirements will be elaborated in section 6 .

\footnotetext{
${ }^{\dagger}$ The definition was created by the Joint DOE/DoD Information Barriers Working Group. See Wolford et al., Software Authentication, UCRL-JC-144254, Lawrence Livermore National Laboratory (2001).
} 
4. "Will the method disclose the presence of high-explosive (HE) in a measured item?"

Not HE specifically, however the measurements are sensitive to the amount of low atomic mass material shielding the detector from the source of neutrons. Thus they could provide a consistency check on other measurements used to show the presence or absence of HE.

5. "What are the prospects for creating a warhead authentication system based on the neutron correlation method using existing technology?"

Existing detector systems based on ${ }^{3} \mathrm{He}$ proportional counters, short dead-time electronics, and custom-designed analysis units would be adequate for demonstration of Feynman Moment neutron correlation analysis techniques for warhead authentication in the near term. Foreseen applications would require detector modifications to increase efficiency and incorporate information barriers.

\section{NEUTRON SOURCES AND CORRELATION: A BRIEF REVIEW}

\subsection{Overview}

Neutrons are produced by various nuclear reactions. The number of neutrons emitted from a single reaction is referred to as the multiplicity, $v$, and is a function of isotope and incident particle energy (as applicable). To illustrate, the multiplicities of a few reactions are provided in Table 1 below.

Table 2.1. Examples of Selected Reactions and their Multiplicities

\begin{tabular}{|c|c|c|}
\hline Reaction & Example & Multiplicity $(v)$ \\
\hline Fission & ${ }^{240} \mathrm{Pu}$ spontaneous & 2.15 \\
& ${ }^{252} \mathrm{Cf}$ spontaneous & 3.86 \\
& ${ }^{239} \mathrm{Pu}(\mathrm{n}, \mathrm{fis})$ & 3.86 \\
& ${ }^{235} \mathrm{U}(\mathrm{n}, \mathrm{fis})$ & 3.86 \\
\hline Induced non-fission & $(\alpha, \mathrm{n})$ impurity reaction & $1=$ Poisson \\
& $(\mathrm{n}, 2 \mathrm{n})$ threshold reaction & 2 \\
& spallation & varies \\
\hline
\end{tabular}

Neutrons emitted simultaneously ${ }^{\dagger}$ from a single reaction are correlated in time, but detecting such correlation becomes more difficult as scattering collisions introduce randomness into the neutron's path. Nonetheless, measurements made under certain circumstances can still reflect the initial correlation of the particles, and time-resolved measurements of neutrons from a correlated source can exhibit intervals between counts that are not purely random. That is, as a group their distribution in time will differ from predictions based on Poisson statistics. Statistical analysis of a large set of such intervals reveals the nature of the correlation of the source and its deviation from purely random neutron emission.

Another important phenomenon is neutron multiplication, which occurs when neutrons generated in (or entering) some medium induce subsequent fissions, which themselves can lead to additional fissions and produce a "chain". In a typical multiplying medium, the period between induced fissions can be as short as $1 \mathrm{~ns}$, giving effectively simultaneous (or highly correlated) neutron production from multiple fissions. This yields neutrons that appear in "bursts" that are less frequent, but more intense, than a random source with the same effective neutron intensity.

Combined, these processes-spontaneous neutron emission with associated multiplicity, induced multiplication, scattering, moderation, and absorption - affect the nature of correlations in neutron counts. The purpose of neutron correlation analysis techniques is to use statistical analysis of time-tagged measurements to derive estimates of source and material properties based on what is know or assumed about the neutron source, the immediate environment, and the detectors.

\section{DESCRIPTION OF THE METHOD}

\subsection{Approach}

\footnotetext{
${ }^{\dagger}$ Simultaneous, in this case, means within a few nanoseconds $\left(10^{-9} \mathrm{~s}\right)$ of each other, well below the resolving time of the fastest detector systems.
} 
To assess neutron correlation for warhead authentication we used Monte Carlo codes to simulate representative warhead authentication conditions (in terms of neutron source, container, and detector) and then performed statistical analysis of the results. We created new computer modeling capabilities for this purpose and tested them for validity. We have updated nuclear data libraries for neutron multiplicity in plutonium $(\mathrm{Pu})$, and we have developed methods and criteria to apply to our simulated results in evaluating the neutron correlation technique.

\subsection{Application of a Correlation Analysis Method (The Extended Feynman Moment Technique)}

Each Monte Carlo simulation results in a "time-tagged" list of detection events that, when analyzed for correlations in time, yields insight into the composition and configuration of the source-detector system. The fundamental analysis method is that of Feynman as extended at LLNL by Prasad and Snyderman and incorporated by Perkins into the NMAC code. ${ }^{1}$ We refer to the augmented method as the Extended Feynman Moment Technique (EFMT).

Neutron correlation analysis relies on statistics, and therefore must be applied to a large number of independent measurements. One way to achieve this is to consider a single experiment as being made up of many small measurements. For instance, one-second of collected data can be analyzed as 1000 measurements of duration $1 \mathrm{~ms}$ each, or 1,000,000 of duration $1 \mu \mathrm{s}$ each. Separating results from a single "long" measurement into numerous "short" timeintervals is a basic aspect of the Extended Feynman Moment Technique. Histogram analysis is applied to these individual measurements, yielding count distributions for given time-gate widths. Analysis of the frequency distributions across all time gates yields estimates of the average count rate, the second moment (doubles multiplicity), and the third moment (triples multiplicity). These estimates are derived directly from measured (or simulated) results, and do not require any assumptions about the system, its material composition, or configuration.

While multiplicity analysis methods such as passive Neutron Multiplicity Counting (NMC) have been used extensively in the past, there is a notable advantage to EFMT over standard NMC - use of a sliding "gate width" allows direct measurement of the detection system time constant (or what is referred to in NMC terminology as the "die-away" of the Rossi- $\alpha$ distribution) and more accurate and robust prediction of factorial moments. ${ }^{2}$

There are important advantages for warhead authentication to having a measure of the time-constant of the detector system and a high confidence estimate of the asymptotic value of the factorial moment. In particular, the detection time constant proves to be an invaluable measured quantity, describing the nature of the contained and intervening material. It reflects the averaged elapsed time from the emission of a source neutron to its detection, and is a gauge of the effect of scattering or moderating materials in the system. Increased time constants indicate the addition of moderating material to the detection system, and therefore also correspond to correlated changes in detection efficiency and system multiplication. In our authentication approach, the detection time constant is used directly as an indicator of authenticity, in combination with other measured properties, and will be discussed in later sections.

In what may be described as a special case of EFMT, standard NMC uses a single fixed-width time gate, so that the measured moment is based on the assumption that the gate is sufficiently wide to yield a converged or "asymptotic" value. This assumption may be valid for many detection cases, but poor for others, especially when an uncertain amount of moderating material also makes uncertain the characteristic time constant of the detector system. With EFMT, however, counts are analyzed in gates of continuously increasing width (in this case ranging in from $1 \mu \mathrm{s}$ to $512 \mu \mathrm{s}$ ), yielding 512 different count distributions and moments. The 512 measured distributions form points on a fitted-curve that approaches (or can be extrapolated to) the asymptotic value of the factorial moment, and exhibits the time characteristics of the detector system. Thus with EFMT the time constant of the detector system is measured directly, and the derived factorial moments are known with greater confidence and robustness than in NMC.

The EFMT approach collects more information than standard NMC and is better suited for passive analysis of systems with low source intensity and high multiplication (such as highly enriched uranium, or HEU) for which much of the distinctive physics is embodied in the low probability, high neutron multiplicity, fission chain events. Analysis of HEU typically requires measurement of factorial moments of high order (greater than third order). While it is possible to derive high-order factorial moments from NMC data, such efforts are uniformly hampered by high uncertainty due to sparse data. New techniques developed at LLNL by Prasad and Snyderman obviate the need to calculate high-order moments, by performing least-squares fits of nuclear source and fission chain data to the EFMT sliding-gate distributions. Recent work at LLNL by Nakae and Snyderman shows great promise for the passive characterization of containerized HEU. More information on the application of EFMT to passive analysis of plutonium and HEU can be found in the referenced work by Prasad and Snyderman [1], and has been implemented in the LLNL code BigFit by 
Prasad and Snyderman, Although most of these techniques are beyond the scope of this paper, they could be valuable for future warhead authentication efforts.

Note that EFMT analysis assumes a point-source diffusion model for the neutron flux. This is an inherent limitation to the method that restricts it to source geometries reasonably represented by point sources in diffusing media (in which scattering collisions greatly outnumber neutron absorption reactions). Based on the measurement geometries we've observed in other candidate arms control measurements, the point source approximation is reasonable. Reference measurements could help establish a margin of confidence in this regard.

\subsubsection{Some Count Distribution Examples.}

Multiple measurements in a single gate yield distribution characteristics of the source and detector system. The count distributions are integral to the analysis, and are helpful for illustrating the phenomena of interest. As a basis for comparison, consider the case of a purely uncorrelated (random) neutron source for which the interval between neutron emissions is sampled from a Poisson distribution. The simulated occurrence histogram for any time-gate should agree with that predicted by Poisson statistics (within Monte Carlo statistical uncertainty). The count distribution for the 512 $\mu$ s time gate is shown in Figure 3.1 below for the case of a 1-D model of a point random source in a $4 \pi^{3} \mathrm{He}$ detector. The results for the random source in a non-multiplying medium show good agreement with Poisson statistics.

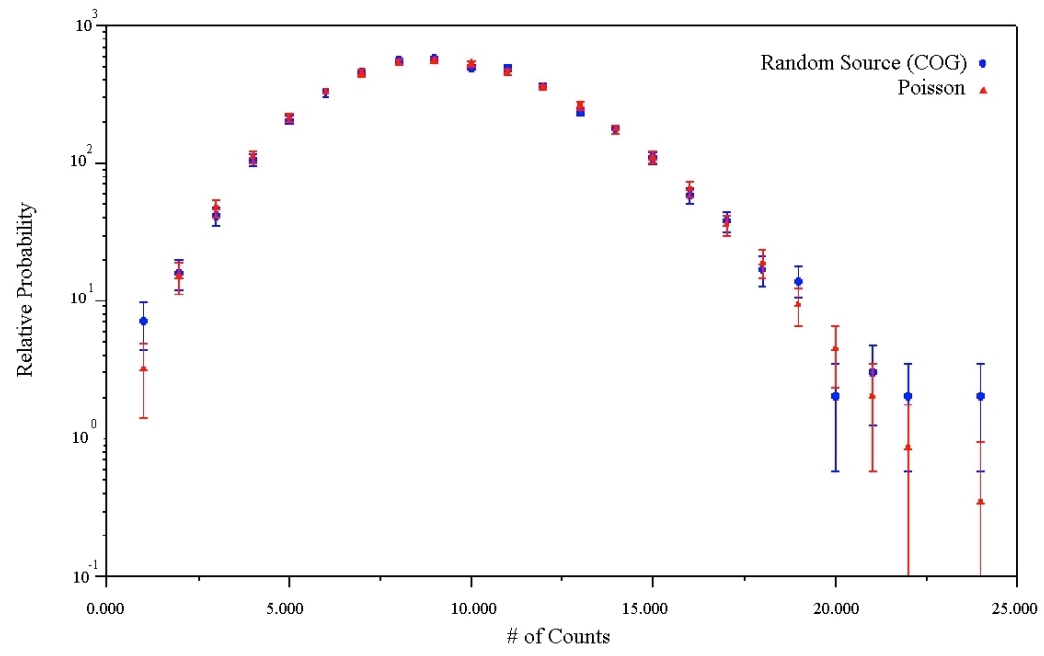

Figure 3.1. NMAC Count distribution for Monte Carlo model of Poisson-distributed neutron source (blue) and analytical Poisson count distribution (red).

Now consider the same model, but with a correlated point source of ${ }^{252} \mathrm{Cf}$ rather than a random source. Recall that $\bar{v}=3.86$ for ${ }^{252} \mathrm{Cf}$. This inherent correlation is manifested as a broadening of the count distribution relative to the theoretical Poisson-based histogram, as shown in Figure 3.2 below. The correlation also increases the "tail" of the distribution, and decreases its peak. 


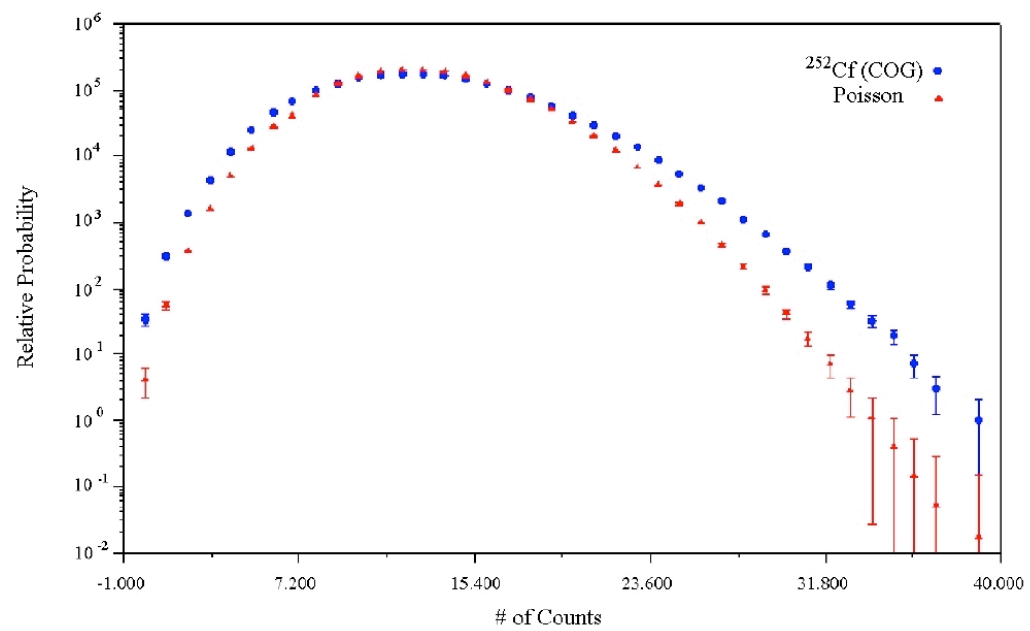

Figure 3.2. NMAC Count distribution for Monte Carlo model of ${ }^{252} \mathrm{Cf}$ neutron source (blue) and analytical Poisson count distribution (red). Neutron multiplicity for ${ }^{252} \mathrm{Cf}$ spontaneous fission is 3.86 .

Finally, consider a correlated source in a multiplying medium, for example a ${ }^{240} \mathrm{Pu}$ spontaneous fission neutron source distributed in ${ }^{239} \mathrm{Pu}$ metal, in isotopic ratios consistent with weapons-grade material. Figure 3.3 shows results for the same detector model as in the last two examples. The effect of induced fission chains is to increase the probability of effectively instantaneous, high-multiplicity neutron emission. The count distribution shows an extended high-end tail, many orders of magnitude more intense than predicted for a Poisson-distributed-source.

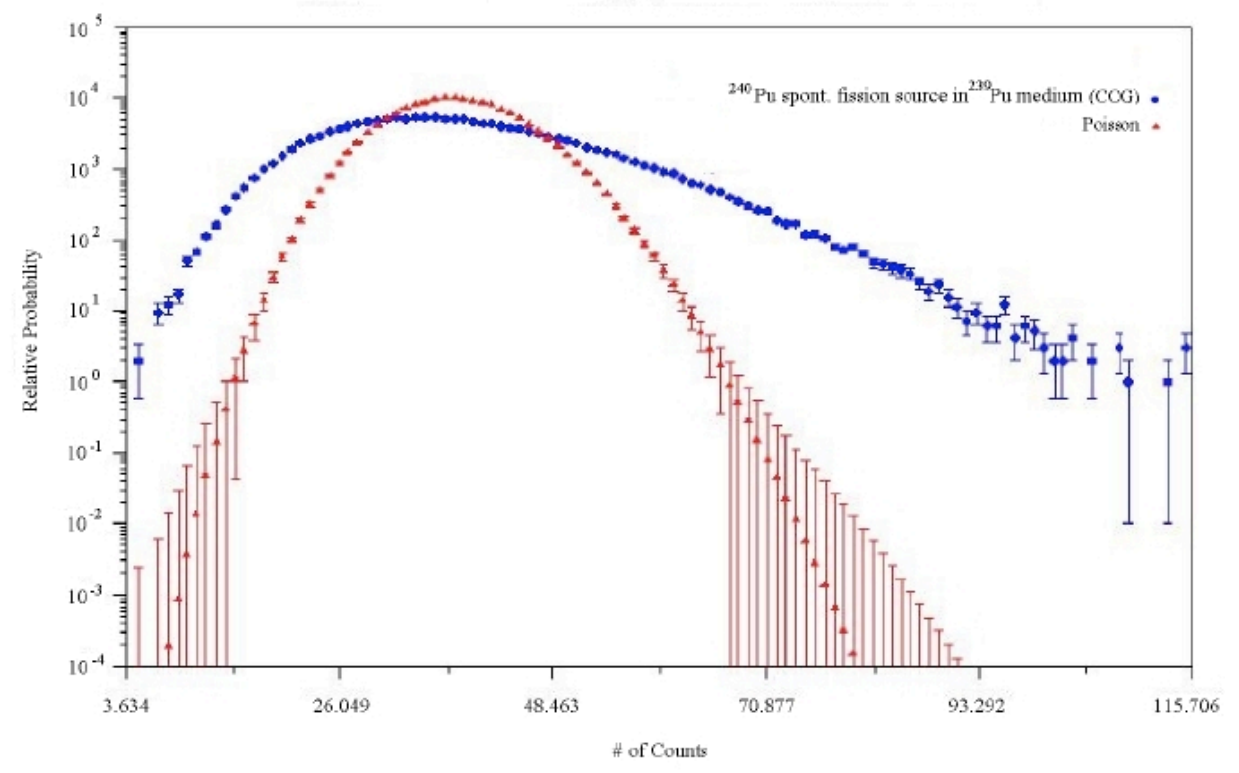

Figure 3.3. NMAC Count distribution for Monte Carlo model of ${ }^{240} \mathrm{Pu}$ neutron source distributed in ${ }^{239} \mathrm{Pu}$ multiplying medium. Multiplication $M=6$ (blue) and analytical Poisson count distribution (red).

3.2.2. Use of EFMT to Determine Material Properties.

Once the count distributions are measured or simulated, then the multiplication $(\mathrm{M})$, detection efficiency $(\varepsilon)$, and spontaneous fission source intensity (S) can be estimated. This requires additional knowledge or assumptions regarding the neutron source, in particular: 
1. Neutron source intensity due to impurity $(\alpha, n)$;

2. Nuclear cross section and multiplicity data for the assumed material composition of the source (nuclear data for the first three factorial moments of the spontaneous fission source),

For all the simulations presented in this report, the $(\alpha, n)$ source rate has been defined to be 0 . Moreover, because the contents of a container are unknown for an actual authentication measurement, applying the proper set of nuclear data to the measured time-tag list is one means of assessing the validity of the assertion that a container holds a warhead or its components. Broadly, we ask, "Are the source's neutron characteristics consistent with it being made of warheadmaterial?" Specifically, "Which set of nuclear data associated with some composition of source materials yields physically acceptable results after EFMT analysis, and do these materials suggest that the source is a warhead-item?" Our ability to answer these questions is addressed in Section 4.

\subsection{Modeling Specifications}

In this section we describe the Monte Carlo model used to evaluate the application of neutron multiplicity counting for warhead authentication.

\subsubsection{Monte Carlo Code.}

We used the LLNL Monte Carlo code COG (versions 10.17a and 10.19nu) ${ }^{3}$ for modeling warhead authentication measurements. The simulations incorporate all the relevant physical phenomena of the source, container, and detectors, such as:

- Spontaneous fission rate and spectrum (function of isotope)

- Spontaneous fission multiplicity (function of isotope, incident neutron energy ${ }^{*}$ )

- Induced fission multiplicity (function of isotope, incident neutron energy)

- Induced fission spectrum (function of isotope, incident energy)

- Neutron transport (scattering, absorption and other reactions), including time-tag information

Most of the physics is implemented in the form of evaluated nuclear data libraries, with use of analytical approximations limited to the multiplicity distributions for uranium, for which no data was readily available.

\subsubsection{Modeled Neutron Sources and Multiplying Media.}

The plutonium source used for modeling is based on an unclassified specification in the International Handbook of Evaluated Criticality Safety Benchmark Experiments, published by the Nuclear Energy Agency ${ }^{4}$. For modeling purposes, the source is taken to be a metal spherical shell based on the PMF022 design in the NEA report. The isotopic composition is as detailed in the NEA report, but the dimensions have been changed slightly to reduce material multiplication and neutron source intensity. Simulated measurements have been performed for the metal, surrounded by various thicknesses of polyethylene (including a bare configuration).

Other non-plutonium, non-warhead-item sources have been defined for modeling purposes. The first is a point source of

${ }^{252} \mathrm{Cf}$ spontaneous fission neutrons. The second is a ${ }^{252} \mathrm{Cf}$ point source inside a spherical shell of polyethylene-reflected $20 \%$ enriched uranium (referred to as LEU). This source poses a more difficult challenge for the proposed authentication systems since it resembles a low-multiplication plutonium source. The ${ }^{252} \mathrm{Cf}$ provides the gross neutron intensity of a plutonium warhead-item source, while the reflected LEU provides the multiplying medium. This source is illustrated in Figure 3.4, below, with details provided in Table 3.1.

\footnotetext{
${ }^{*}$ We consider only neutron-induced fission in this work.
} 


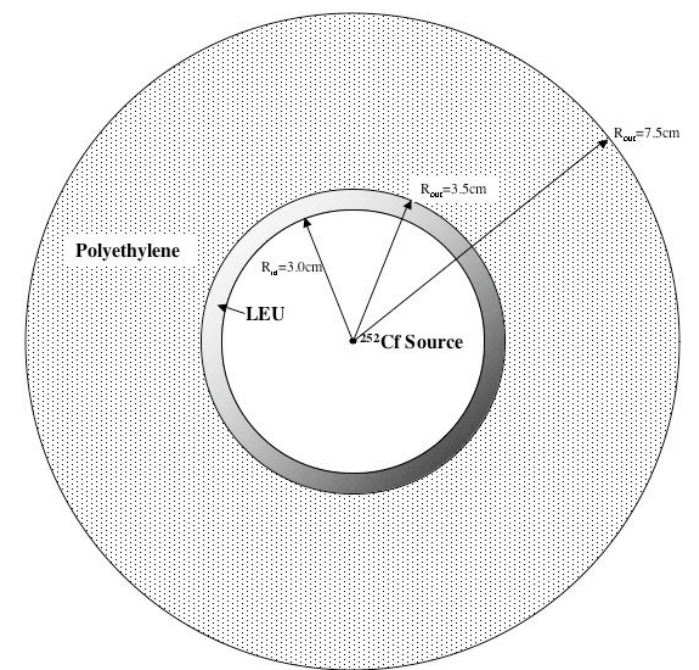

Fig 3.4. Image of LEU spoof source, showing ${ }^{252}$ Cf point source inside of Low-

Enriched Uranium shell wit outer layer of polyethylene.

Table 3.1. Description of neutron sources specified for Monte Carlo model.

\begin{tabular}{|c|c|c|c|c|c|c|c|c|}
\hline $\begin{array}{l}\text { Source } \\
\text { Number }\end{array}$ & $\begin{array}{l}\text { Source or } \\
\text { Component }\end{array}$ & Description & $\begin{array}{l}\text { Dimensions } \\
(\mathrm{cm})\end{array}$ & Mass (g) & $\begin{array}{l}\text { Isotop } \\
\text { Comp } \\
\text { (wt.\% }\end{array}$ & c & $\begin{array}{l}\text { Source Intensity } \mathrm{n} \\
\mathrm{s}^{-1}(\text { based on } 1000 \\
\left.\mathrm{ng}^{-1} \mathrm{~s}^{-1}\right)\end{array}$ & $\mathrm{M}$ \\
\hline Published & Component of & Weapons-grade & $\mathrm{R}_{\text {in }} 6.0$ & $5,813.0$ & ${ }^{239} \mathrm{Pu}$ & 96.41 & 102,900 & 3.15 \\
\hline Specs. & critical assembly & plutonium metal & $\mathrm{R}_{\text {out }} 6.75$ & & ${ }^{240} \mathrm{Pu}$ & 01.77 & & \\
\hline & & & & & $\mathrm{Ga}$ & 01.69 & & \\
\hline 1 & Modified PMF022 & Weapons-grade & $\mathrm{R}_{\text {in }} 6.6$ & $1,273.3$ & ${ }^{239} \mathrm{Pu}$ & 96.41 & 22,500 & 1.11 \\
\hline & layer 6 & $\begin{array}{l}\text { plutonium metal } \\
\text { spherical shell }\end{array}$ & $R_{\text {out }} 6.75$ & & ${ }^{240} \mathrm{Pu}$ & 01.77 & & \\
\hline & & & & & $\mathrm{Ga}$ & 01.69 & & \\
\hline 2 & Californium & Point source & - & - & ${ }^{252} \mathrm{Cf}$ & 100.0 & 22,500 & - \\
\hline 3 & Low-enriched & Point ${ }^{252} \mathrm{Cf}$ inside & Metal: & $1,256.8$ & ${ }^{238} \mathrm{U}$ & 80.0 & 22,500 & 1.28 \\
\hline & $\begin{array}{l}\text { uranium (LEU) } \\
\text { spoof source }\end{array}$ & $\begin{array}{l}\text { LEU shell, with } 4 \\
\mathrm{~cm} \text { polvethylene }\end{array}$ & $\mathrm{R}_{\text {in }} \quad 3.0$ & & ${ }^{235} \mathrm{U}$ & 20.0 & & \\
\hline & (Spoof_LEU_cm4) & shell outside LEU & $\mathrm{R}_{\text {out }} 3.5$ & & & & & \\
\hline & & & Polyethylene & & $\mathrm{H}$ & 14.4 & & \\
\hline & & & $\mathrm{R}_{\text {in }} \quad 3.5$ & & $\mathrm{C}$ & 85.6 & & \\
\hline & & & $\mathrm{R}_{\text {out }} 7.5$ & & & & & \\
\hline
\end{tabular}

Note that the neutron source intensity used for each model-22,500 $\mathrm{s}^{-1}$ - is relatively low and corresponds to approximately $0.59 \mathrm{~kg}$ weapons-grade plutonium, assuming $4 \%$ by weight ${ }^{240} \mathrm{Pu}$ content and a $950 \mathrm{~g} \mathrm{~g}^{-1} \mathrm{~s}^{-1}$ specific neutron intensity. This intensity is predetermined as a result of reducing the multiplication of the original critical assembly component (by increasing the inner radius). The corresponding mass of $0.59 \mathrm{~kg}$ is not meant in any way to be representative of warheads.

\subsubsection{Modeled Container and Source Configuration.}

The neutron sources are placed inside a modified AT-400R style container, based on the container described by Baker and Belooussov ${ }^{5}$. The container defined for our Monte Carlo models has a smaller diameter than specified by Baker, and 
has been simplified by omitting the inner capsules, thermal bridges, aluminum inserts, borated polypropylene inserts, and polypropylene fill. Model dimensions and specifications for the simplified container are provided in Table 3.2 below.

Table 3.2. Description of the material container specified for Monte Carlo model.

\begin{tabular}{|ll|}
\hline Container Characteristic & Specification $(\mathrm{cm})$ \\
\hline Outer Radius & 35.3 \\
Outer steel thickness & 0.3 \\
Outer polyurethane fill thickness & 9.0 \\
Inner steel shell thickness & 0.4 \\
Lid and bottom & Steel, 0.3 \\
& Polyurethane, 9.0 \\
Total height, with lid and bottom & 140.6 \\
Usable Dimensions and Volume & Radius 25.6 \\
& Height 120.9 \\
& $2.5 \times 10^{5} \mathrm{~cm}^{3}$ \\
\hline
\end{tabular}

The center of the spherical source is on the vertical axis of the container, at an elevation $33 \mathrm{~cm}$ above floor level, colinear with the centers of the pods. The container and source are illustrated in Figure 3.5, below.

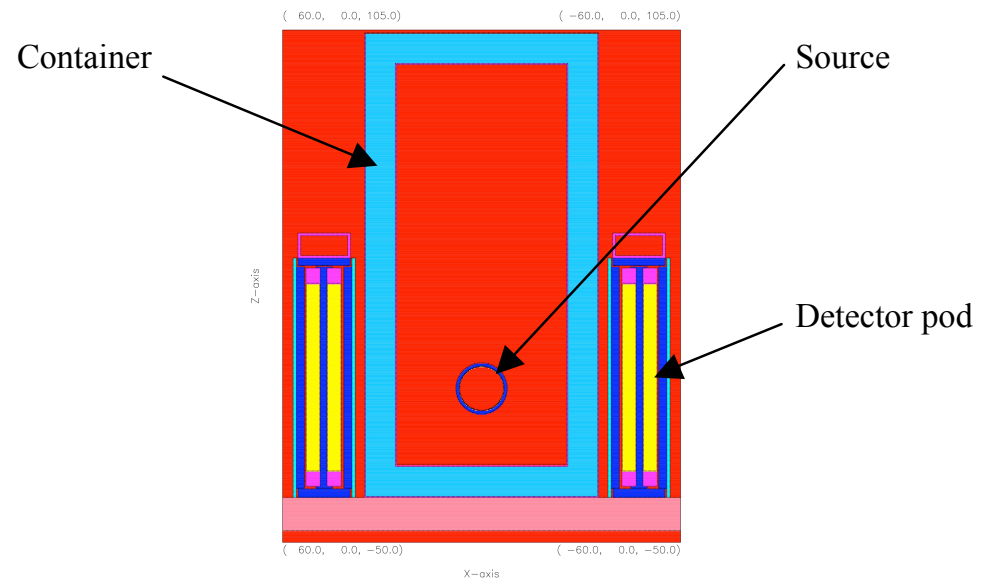

Figure 3.5. Vertical bisection picture of the warheaditem source, container, and detector pods (aluminum floor also visible).

\subsubsection{Modeled Detectors.}

The detector units, or "pods" as they will be referred to, are modeled to closely match pods developed at LLNL for multiplicity counting purposes. They consist of 14 proportional counter neutron detectors with ${ }^{3} \mathrm{He}$ fill gas at 2 atmospheres pressure. The detectors are supported by polyethylene spacers and structural material, with additional polyethylene added to some surfaces for increased neutron moderation. The pod is covered with a padded case. The electronics for the detectors are housed in a stainless steel box affixed to the top of the pod. The surface of the padded case on each pod is offset from the origin by $38.1 \mathrm{~cm}$, providing a $2.8 \mathrm{~cm}$ gap between the pod and the outer surface of the container. The detector tubes are aligned in two rows with axes parallel to the face. The minimum distance from each axis to the center of the neutron source is $44.45 \mathrm{~cm}$ and $50.80 \mathrm{~cm}$, respectively. Cross-sectional illustrations of the detector pods, container, and source are shown in Figure 3.6 below; perspective images are provided in Figure 3.7. 


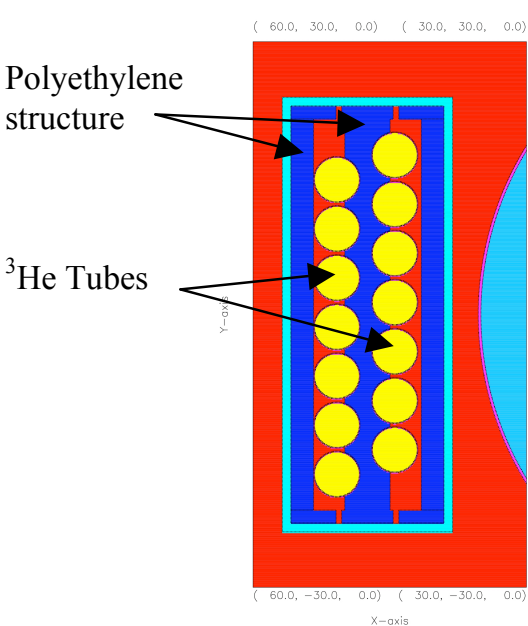

(a)

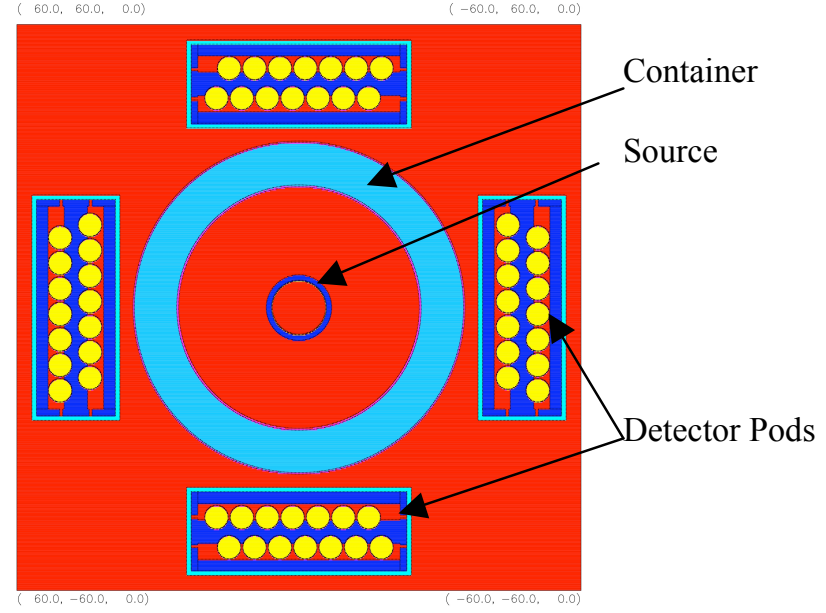

(b)

Figure 3.6. (a) Horizontal cross-section of a single detector pod, showing fourteen ${ }^{3}$ He proportional detector tubes, polyethylene support structure, and outer foam case picture of the warhead-item source, container, and detector pods (portion of container is also visible); (b) Horizontal cross-section of source, container, and fourpod configuration.

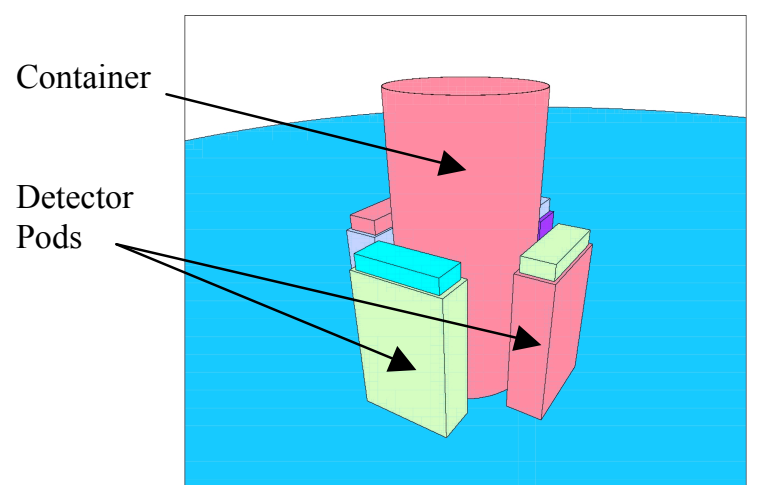

(a)

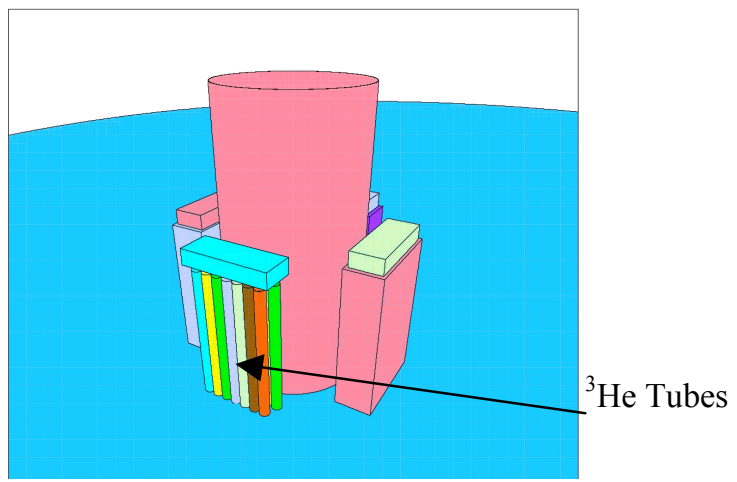

(b)

Figure 3.7. (a) Perspective picture of container and four-pod configuration; (b) Same image with outer-case removed from one pod to show individual ${ }^{3}$ He proportional detector tubes.

\subsubsection{Overall Model Configuration.}

The laboratory environment has been modeled in low detail and is based previous models of the building 262 facility at LLNL that demonstrated the lack of influence of the surroundings. The detectors and container are defined to be on a low-density aluminum floor, representing a grated aluminum floor in the actual facility. The building is modeled as a spherical concrete shell with a concrete dividing wall in the center. Two cross-sectional views of the model-including source, container, pods, and building — are shown in Figure 3.8, below. 


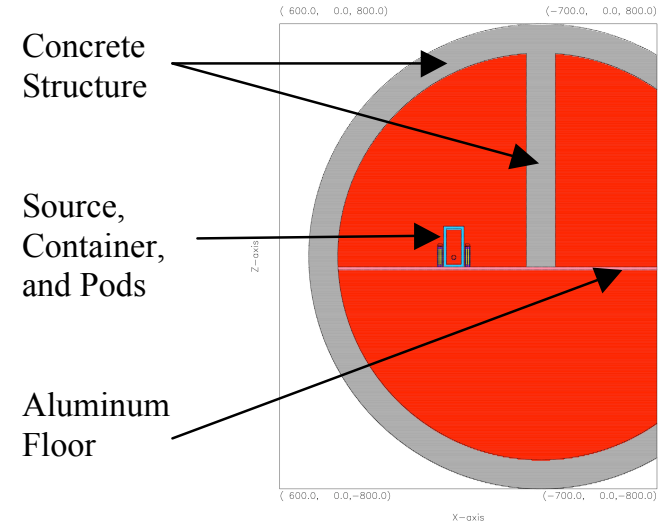

(a)

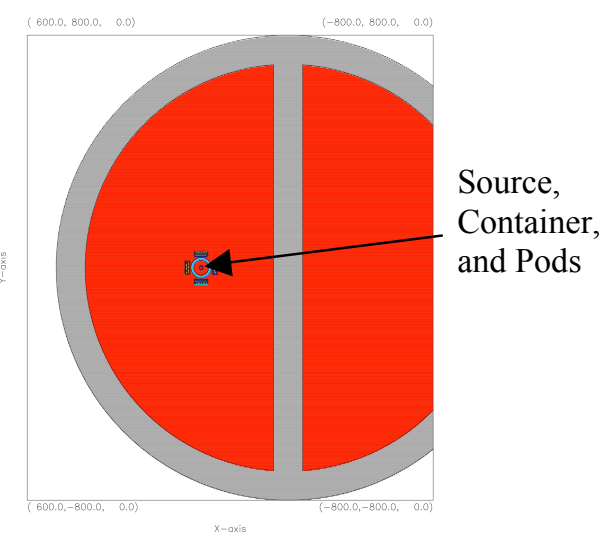

(b)

Figure 3.8. (a) Vertical bisection picture of COG model, showing building, wall, floor, detectors, container, and source; (b) Horizontal bisection picture of COG model showing building, full center wall, four pods, container, and source.

Table 3.3. Description of the laboratory building specified for the Monte Carlo model.

\begin{tabular}{|l|l|l|}
\hline \multicolumn{2}{|c|}{ Compoment } & \multicolumn{1}{c|}{ Geometry } \\
\hline Grated Aluminum Floor: & $\begin{array}{l}\text { Disc: radius } 700 \mathrm{~cm} \\
\text { thickness } 10 \mathrm{~cm}\end{array}$ & $\begin{array}{l}\text { Reduced-density aluminum } \\
\rho=0.5 \mathrm{~g} \mathrm{~cm}^{-3}\end{array}$ \\
\hline Middle Wall: & Slab: $100 \mathrm{~cm} \times 1400 \mathrm{~cm} \mathrm{x} 837 \mathrm{~cm}$ & $\begin{array}{l}\text { LLNL Concrete } \\
\rho=2.25 \mathrm{~g} \mathrm{~cm}^{-3}\end{array}$ \\
\hline Outer Walls: & $\begin{array}{l}\text { Sphere: inner radius, } 700 \mathrm{~cm} \\
\text { thickness } 100 \mathrm{~cm}\end{array}$ & $\begin{array}{l}\text { LLNL Concrete } \\
\rho=2.25 \mathrm{~g} \mathrm{~cm}^{-3}\end{array}$ \\
\hline
\end{tabular}

\section{AN APPROACH TO AUTHENTICATION}

\subsection{General Approach}

We have devised an approach for warhead authentication that uses neutron correlation analysis to assess containerized items with respect to declarations. While a full description of a warhead authentication regime is beyond the scope of this paper, we nevertheless present a concept of how a measurement based on neutron correlation could be applied. This approach assumes that parties to an agreement will specify warhead characteristics that sufficiently define warhead "authenticity", e.g. the presence and amounts of certain materials. The approach, in four steps, is:

1. Characterize and Measure. Make (or simulate) measurements with a detector system for which the detection efficiency for a fission neutron source has been experimentally (computationally) determined.

2. Analyze for Agreement with Warhead Assumptions. Perform EFMT analysis of measured data using set(s) of previously agreed-upon characteristics for acceptable warhead. Data includes nuclear cross-sections, multiplicities, and impurity-source intensities.

3. Analyze for Agreement with Potential Spoofs As required, Perform EFMT analysis of measured data using set(s) of previously agreed-upon characteristics for potential spoof sources.

4. Form conclusions regarding authenticity and/or confidence.

The approach is described in greater detail in the sections that follow.

\subsection{A Parameter Space for Testing the "Warhead Hypothesis"}

In a warhead authentication regime, there will be a range of material compositions, geometries, and configurations that indicate "warheads" or their components. We are not attempting to determine these characteristics, but rather to evaluate 
whether the attributes of the contained materials that are accessible to EFMT are sufficient to test a "Warhead Hypothesis". Accordingly, we will test the following statement:

"The measurement is consistent with the contained item being a warhead or warhead component(s)."

To determine the validity of this assertion, there are two underlying subhypotheses that must be tested. They can be stated as:

Subhypothesis 1. "The measurement is consistent with assumed warhead properties (source, multiplicity, fission)."

Subhypothesis 2. "The measurement is consistent with assumed spoof properties."

To decide if the measured properties of the contained material are more consistent with a warhead or a spoof item, we must specify what properties will be measured and then determine their acceptable ranges and combinations of values. At this point in the discussion, we focus on attributes one can determine through neutron correlation techniques.

\subsubsection{Efficiency-Moderation-Multiplication Parameter Space}

From measurements yielding time-tagged count data, neutron correlation analysis provides the following attributes ${ }^{6}$ :

- $\quad$ Source Strength (S) / Mass,

- Multiplicity (M),

- Detection Efficiency $(\varepsilon)$,

- Moderation Time Constant $\left(\lambda^{-1}\right)$

Based on our analysis, it would not be advisable to use source strength / mass as a single indicator of authenticity, because the range of masses and isotopic compositions can lead to large uncertainties in the derived physical quantities. In general, we expect weapons grade material to have relatively low spontaneous fission neutron intensity, based on the minimum acceptable ${ }^{239} \mathrm{Pu}$ enrichment and the maximum size/weight capacity of the container. However, in the scenarios considered for this study, these limits are actually well above simulated intensities and are thus not useful for discrimination. Rather, derived estimates of mass / source strength are useful primarily for confirmation of results for known masses.

For the remaining three parameters, the best approach for hypothesis testing is to consider them simultaneously so that they define a parameter space, as illustrated in Figure 4.1, below. Viewing the values as points in a parameter space allows ready observation of correlations and trends, and takes full advantage of their observed physical interdependencies.

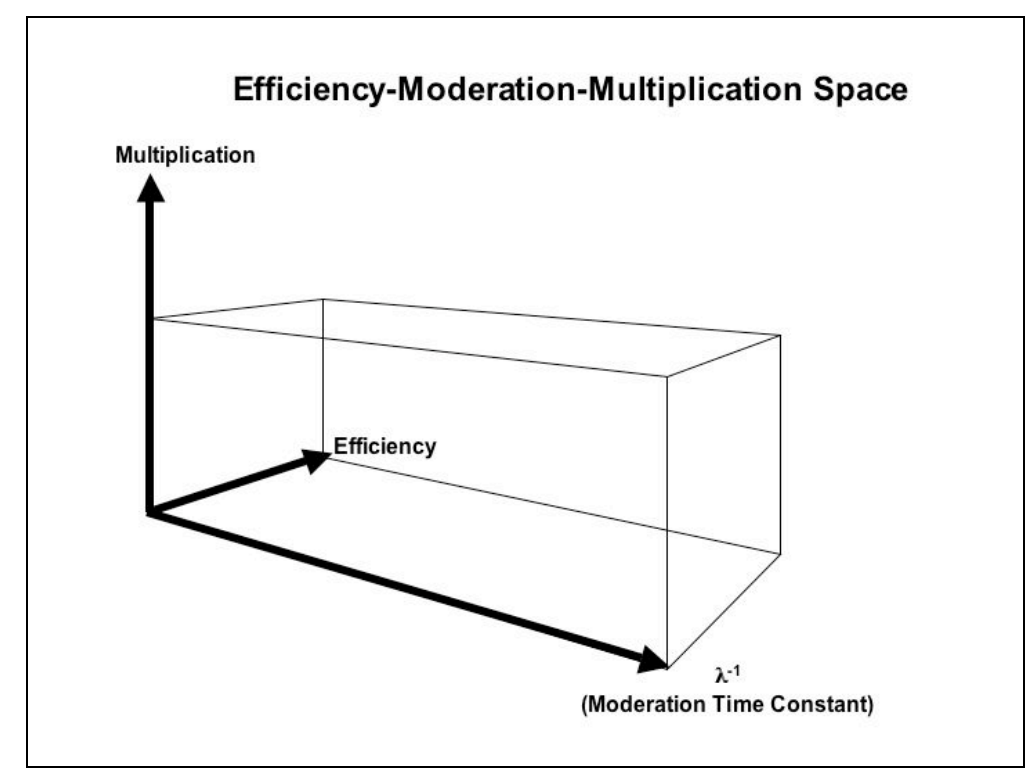

Figure 4.1. Notional representation of the three-parameter space of efficiency (e), moderation $\left(\lambda^{-1}\right)$, and multiplication (M), used for assessing characteristics of measured containerized sources. 
The nature of each of each of the parameters and their relation to each other are discussed in more detail below.

\begin{tabular}{|c|c|}
\hline \multicolumn{2}{|l|}{ Detection Efficiency } \\
\hline Description & $\begin{array}{l}\text { For the particular configuration of pods, source, and container used in modeling, there is a } \\
\text { maximum detection efficiency that can be determined experimentally or through simulation. } \\
\text { While we have not determined the maximum efficiency of the system, COG simulations of a } \\
{ }^{252} \mathrm{Cf} \text { point source in the container show that the system is over-moderated-that is, any } \\
\text { additional moderation added to the system will reduce detection efficiency. Therefore, we } \\
\text { conclude that the maximum efficiency is realized with a bare fission neutron source, and our } \\
\text { simulations show that this maximum efficiency for the four-detector system is approximately } \\
8.5 \% \text {. Thus, allowing for statistical uncertainty, the maximum system efficiency for detection of } \\
\text { fission spectrum neutrons will be defined to be } 9 \% \text { (slight variations in spectral intensity due to } \\
\text { isotopic changes notwithstanding). }\end{array}$ \\
\hline Limits & $\varepsilon<0.09$ \\
\hline $\begin{array}{l}\text { Relation to Other } \\
\text { Parameters }\end{array}$ & Inverse correlation to moderation time constant \\
\hline
\end{tabular}

\begin{tabular}{|ll|}
\hline Moderation & \\
\hline Description & $\begin{array}{l}\text { The time constant, } \lambda^{-1} \text {, has been described in previous sections, and for the detectors modeled } \\
\text { has been determined experimentally by Kerr and Snyderman at roughly } 36 \mu \mathrm{s}^{-1} \text {. The addition of } \\
\text { the polyurethane on the container increases the simulated value to approximately } 40 \mu \mathrm{s}^{-1} \text {. Thus, } \\
\text { for the container we are describing, measured values less } 40 \mu \mathrm{s}^{-1} \text { are non-physical and would } \\
\text { indicate invalid data. The addition of a few grams per square centimeter of moderating material } \\
\text { around the neutron source would further increase the estimated moderation time constant to the } \\
\text { range of } 50 \text { to } 70 \mu \mathrm{s}^{-1} .\end{array}$ \\
$\begin{array}{l}\lambda^{-1},\left(\text { containerized) }>40 \mu \mathrm{s}^{-1}\right. \\
\text { Limits } \\
\begin{array}{l}\text { Relation to Other } \\
\text { Parameters }\end{array}\end{array}$ & $\begin{array}{l}\text { Inverse correlation to efficiency } \\
\end{array}$ \\
\hline
\end{tabular}

\begin{tabular}{|ll|}
\hline Multiplication & \\
\hline Description & $\begin{array}{l}\text { Warheads or warhead components may be packaged in a variety of configurations and } \\
\text { geometries, giving rise to a range of measured multiplication, which an authentication regime } \\
\text { would need to make allowances for. It might be reasonable to require a minimum estimated } \\
\text { value in the range 1.02-1.04 for the entire containerized source, including the effects of } \\
\text { moderation and reflection in the container. Note, however, that while multiplication may be an } \\
\text { effective single indicator of authenticity for some potential warhead materials, it is likely to fail } \\
\text { for other configurations containing non-warhead spoof sources (discussed below). } \\
\text { Multiplication is affected by system moderation, especially when moderating material with } \\
\text { relatively low-absorption cross section is situated outside of a low-M geometry of fissionable } \\
\text { material. In general, additional moderator will increase M for thin-shell multiplying media. }\end{array}$ \\
$\begin{array}{l}\text { Limits } \\
\text { Relation to Other } \\
\text { Parameters }\end{array}$ & Direct correlation to moderation for low-multiplication sources \\
\hline
\end{tabular}




\subsubsection{Hypothesis Testing.}

For this study, we provide a simple example of how the hypothesis testing might be implemented. The following results should not be taken as a general prescription for all warhead authentication scenarios, rather they apply only to the few cases considered. Also note that the values of $\varepsilon, \mathrm{M}$, and $\lambda^{-1}$ cited above as criteria come from our knowledge of specific detectors and our assumptions about container moderation. Any measurement regime based on EFMT would adjust these values for the specific application.

The first step in testing the Warhead Hypothesis is to test subhypothesis 1 by analyzing time-tag data with warhead item assumptions. The derived estimated parameter set $\left(\mathrm{M}, \varepsilon, \lambda^{-1}\right)$ is tested for the following conditions:

1. $\mathrm{M}>1.04$ (or other negotiated minimum multiplication);

2. $\varepsilon<0.09$ (maximum system efficiency, experimentally determined);

3. The parametric point $\left(\mathrm{M}, \varepsilon, \lambda^{-1}\right)$ is within statistical and systematic error of the benchmark warhead-item acceptable region.

If we accept subhypothesis 1, the process is complete and the Warhead Hypothesis is accepted. If subhypothesis 1 is rejected, the data are re-analyzed with non-warhead assumptions (spoof assumptions). Using the spoof assumption, the derived estimated parameter set $(\mathrm{M}, \varepsilon, 1)$ is tested for physicality and acceptability. Specifically, this requires:

1. $\mathrm{M}>1$ (physical result);

2. $\varepsilon<0.09$ (maximum system efficiency, experimentally determined);

3. The parametric point $\left(\mathrm{M}, \varepsilon, \lambda^{-1}\right)$ is within statistical and systematic error of the benchmark spoof-item acceptable region.

If we accept hypothesis 2, the Warhead Hypothesis is rejected. However, if both hypothesis 1 and hypothesis 2 are rejected, no conclusion is reached regarding the Warhead Hypothesis.

The truth table below summarizes these statements.

\begin{tabular}{|ll|}
\hline Subhypothesis $1=$ True & Accept Warhead Hypothesis \\
Subhypothesis $1=$ False & Reject Warhead Hypothesis \\
Subhypothesis $2=$ True & No Conclusion \\
Subhypothesis $1=$ False & \\
Subhypothesis $2=$ False & Disallowed a priori \\
Subhypothesis $1=$ True & \\
Subhypothesis $2=$ True & \\
\hline
\end{tabular}

\subsection{Rationale for Test Sequence}

Note that we accept the Warhead Hypothesis based on a single test, but require two tests for rejection. The reason is that, in some cases, all three attributes $\left(\mathrm{M}, \varepsilon, \lambda^{-1}\right)$ are needed to distinguish warhead-items from spoofs. Because the ${ }^{252} \mathrm{Cf}$ spoofs have, by nature, $\mathrm{M}=1$, only two attributes are available for analysis. With only two "degrees of freedom" the results of warhead and non-warhead analysis become degenerate, yielding inconclusive results. In other words, in many cases the variation in multiplication is necessary to distinguish between warhead and non-warhead items. Acceptance of Subhypothesis 1 (warhead assumptions) is based on all three parameters and is conclusive. In such cases, further validation with a two-parameters test of Subhypothesis 2 (spoof assumptions) is not necessary. 


\section{MODEL RESULTS AND ANALYSIS}

\subsection{Overview}

The results of NMAC analysis for Sources 1, 2, and 3 are discussed in the sections below. All COG time-tagged results have been analyzed using NMAC Version 65.4. We took advantage of option settings within the software to improve the fit to data from the moderating container.

Note that the simulated measurement times range from a few minutes to roughly four hours, depending on the source. In general, highly moderated sources will exhibit lower neutron emission rates (from the container) and will require longer measurement durations for adequate statistics. Recall that the specified source intensity is relatively low, corresponding to roughly $0.59 \mathrm{~kg}$ of weapons-grade $\mathrm{Pu}$. Accordingly, our estimates of the time required for each measurement are conservative. The effect on analysis statistics and required measurement time of applying EFMT to more intense spontaneous fission sources has not been investigated in detail, but should be addressed in any subsequent work.

Our conclusion for all analyses performed is that the "Warhead Hypothesis" can be consistently and confidently rejected for the non-Pu "spoof" sources. Details of how we reached this conclusion are provided below.

\subsection{Results of Hypothesis Testing}

\subsubsection{Benchmark Warhead-Item Simulation.}

Results of analysis of the warhead-item (Source 1) using correct assumptions-what we refer to as the "Benchmark Case"- are displayed in Figure 5.1 and tabulated in Table 5.1. The source has been modeled with different thicknesses of polyethylene around the plutonium metal.
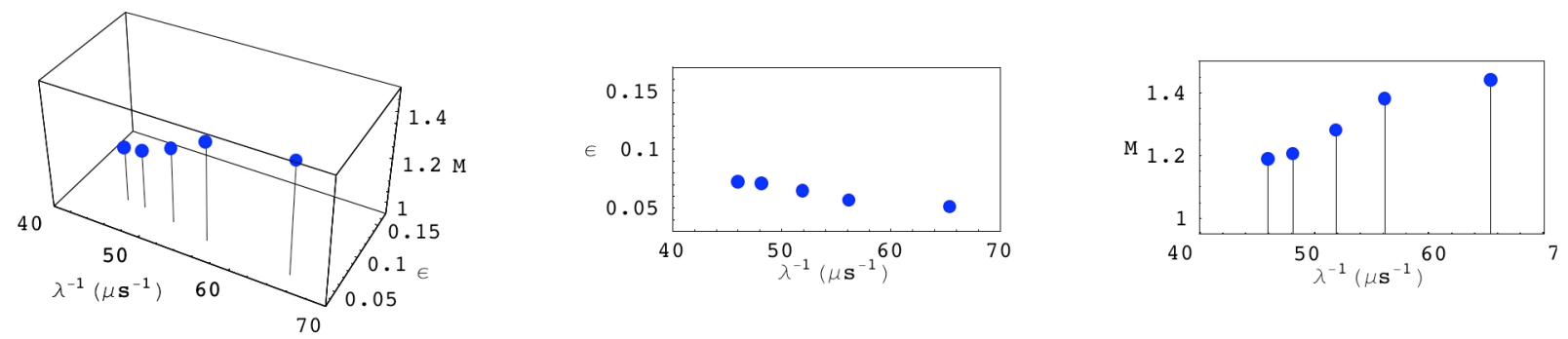

Figure 5.1. Estimated parameters $\lambda^{-1}$, E, and M for Source-1 (modified NEA plutonium subcritical assembly component), based on assumed ${ }^{240} \mathrm{Pu}$ spontaneous fission source and ${ }^{239} \mathrm{Pu}$ multiplying medium. Results are shown for increasing thicknesses of spherical polyethylene shell $\left(0.94 \mathrm{~g} \mathrm{~cm}^{-3}\right.$ ) around the plutonium metal (with increasing $\lambda^{-1}$, results are for $0,1,2,3$, and $4 \mathrm{~cm}$ polyethylene, respectively).

Table 5.1. Results of NMAC analysis of COG simulations. Red indicates non-physical results. Blue indicates physical results that are not individually consistent with a warhead-item source.

\begin{tabular}{|c|c|c|c|c|c|c|c|c|}
\hline & $\begin{array}{l}\text { Time } \\
\text { constant } \\
\lambda\left(\mu \mathrm{s}^{-1}\right)\end{array}$ & $\begin{array}{ll}\sigma & \lambda \\
\left(\mu s^{-1}\right)\end{array}$ & Effic. $\varepsilon$ & $\sigma \varepsilon$ & $\begin{array}{l}\text { Multipication } \\
\text { M }\end{array}$ & $\sigma \mathbf{M}$ & $\begin{array}{l}\text { Duration } \\
\text { (minutes) }\end{array}$ & Mass \\
\hline \multicolumn{9}{|c|}{$\begin{array}{l}\text { Warhead-items with warhead- } \\
\text { assumptions ("Benchmark") }\end{array}$} \\
\hline \multicolumn{9}{|l|}{ 1. Warhead-item (Source 1) } \\
\hline Bare & 46.00 & 0.22 & 0.072 & 0.00023 & 1.19 & 0.0016 & 72.6 & 24.59 \\
\hline Polyethylene, $1 \mathrm{~cm}$ & 48.16 & 0.22 & 0.071 & 0.00023 & 1.21 & 0.0002 & 72.6 & 24.34 \\
\hline Polyethylene, $2 \mathrm{~cm}$ & 51.91 & 0.16 & 0.064 & 0.00017 & 1.28 & 0.0010 & 145.0 & 24.72 \\
\hline Polyethylene, $3 \mathrm{~cm}$ & 56.15 & 0.16 & 0.056 & 0.00018 & 1.38 & 0.0019 & 144.7 & 25.50 \\
\hline Polyethylene, $4 \mathrm{~cm}$ & 65.39 & 0.14 & 0.051 & 0.00015 & 1.44 & 0.0018 & 232.6 & 25.26 \\
\hline
\end{tabular}




\subsubsection{Spoof Source Analyses.}

Next, we consider analysis of data from a non-warhead sources intended to spoof the detection system. The first spoof is the ${ }^{252} \mathrm{Cf}$ point source (Source 2). Following the procedure described above, the first step is to conduct EFMT analysis of the data, assuming the modeled object is a valid warhead-item. In this case, we assume it is a ${ }^{240} \mathrm{Pu}$ spontaneous fission source within a ${ }^{239} \mathrm{Pu}$ multiplying medium. The results for the californium source are shown in Table 5.2, and in red in Figure 5.2, along with the Source-1 warhead-item results. The ${ }^{252} \mathrm{Cf}$ source has been modeled with polyethylene spheres of different thickness, with the point source at their center.

Table 5.2. Results of NMAC analysis of COG simulations. Red indicates non-physical results, while blue indicates physical results that are not individually consistent with a warhead-item source.

\begin{tabular}{|c|c|c|c|c|c|c|c|c|}
\hline & $\begin{array}{l}\text { Time } \\
\text { constant } \\
\lambda\left(\mu \mathrm{s}^{-1}\right)\end{array}$ & $\begin{array}{ll}\sigma & \lambda \\
\left(\mu s^{-1}\right)\end{array}$ & Effic. $\varepsilon$ & $\sigma \varepsilon$ & $\begin{array}{l}\text { Multipication } \\
\text { M }\end{array}$ & $\boldsymbol{\sigma} \mathbf{M}$ & $\begin{array}{l}\text { Duration } \\
\text { (minutes) }\end{array}$ & Mass \\
\hline \multicolumn{9}{|c|}{ Spoofs with spoof- assumptions } \\
\hline \multicolumn{9}{|l|}{ 2. ${ }^{252} \mathrm{Cf}$ (Source 2$)$} \\
\hline Bare & 44.32 & 0.16 & 0.083 & 0.00016 & 1 & 0 & 74.5 & $9.92 \mathrm{E}-9$ \\
\hline Polyethylene, $1 \mathrm{~cm}$ & 46.46 & 0.5 & 0.084 & 0.00015 & 1 & 0 & 7.6 & $9.37 \mathrm{E}-9$ \\
\hline Polyethylene, $2 \mathrm{~cm}$ & 45.7 & 0.54 & 0.077 & 0.00015 & 1 & 0 & 7.5 & $9.65 \mathrm{E}-9$ \\
\hline Polyethylene, $4 \mathrm{~cm}$ & 51.91 & 0.21 & 0.063 & 0.00005 & 1 & 0 & 74.3 & $9.70 \mathrm{E}-9$ \\
\hline Polyethylene, $8 \mathrm{~cm}$ & 60.93 & 0.387 & 0.034 & 0.00005 & 1 & 0 & 74.2 & 9.96E-9 \\
\hline \multicolumn{9}{|l|}{ 3. LEU Spoof (Source 3) } \\
\hline Bare & 45.64 & 0.15 & 0.083 & 0.0002 & 1.07 & 0.0015 & 74.3 & $9.70 \mathrm{E}-9$ \\
\hline Polyethylene, $4 \mathrm{~cm}$ & 59.3 & 0.11 & 0.059 & 0.0001 & 1.17 & 0.0013 & 233.8 & 9.70E-9 \\
\hline \multicolumn{9}{|l|}{$\begin{array}{l}\text { Spoofs with warhead- } \\
\text { assumption }\end{array}$} \\
\hline \multicolumn{9}{|l|}{ 4. ${ }^{252} \mathrm{Cf}$ (Source 2$)$} \\
\hline Bare & 44.32 & 0.16 & 0.150 & 0.0002 & 1.003 & 0.0005 & 72.6 & 13.24 \\
\hline Polyethylene, $1 \mathrm{~cm}$ & 46.46 & 0.51 & 0.140 & 0.0007 & 1.031 & 0.0020 & 72.6 & 13.50 \\
\hline Polyethylene, $2 \mathrm{~cm}$ & 45.69 & 0.54 & 0.143 & 0.00062 & 0.979 & 0.0014 & 145.0 & 12.96 \\
\hline Polyethylene, $4 \mathrm{~cm}$ & 51.91 & 0.21 & 0.114 & 0.0002 & 0.992 & 0.0007 & 144.7 & 13.28 \\
\hline Polyethylene, $8 \mathrm{~cm}$ & 60.3 & 0.387 & 0.059 & 0.0002 & 1.010 & 0.0016 & 232.6 & 13.96 \\
\hline \multicolumn{9}{|l|}{ 5. LEU Spoof (Source 3) } \\
\hline Bare & 45.64 & 0.15 & 0.150 & 0.00021 & 1.030 & 0.0005 & 74.3 & 13.37 \\
\hline Polyethylene, $4 \mathrm{~cm}$ & 59.3 & 0.111 & 0.112 & 0.00013 & 1.066 & 0.0005 & 233.8 & 13.20 \\
\hline
\end{tabular}

The parametric differences between the two sources are drastic. For the spoof source, the predicted efficiency is nonphysical (too high) for time-constants below roughly $60 \mu \mathrm{s}^{-1}$, and the estimated multiplication is far below expectations for all values of $\lambda^{-1}$.
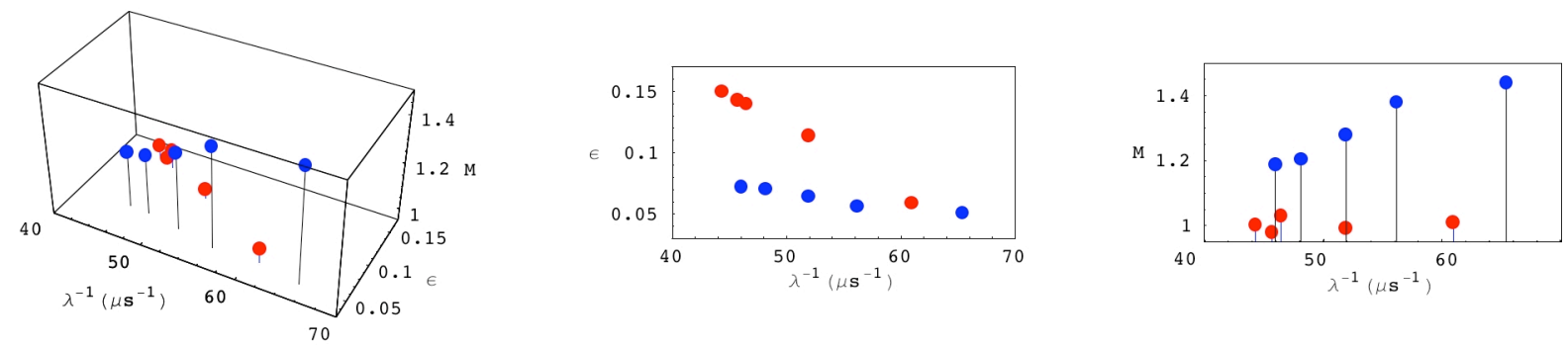

Figure 5.2. Estimated parameters $\lambda^{-1}, E$, and $M$ for Source-2 $\left({ }^{252} C f\right.$ point source at the center of polyethylene sphere, for various sphere thicknesses), based on an assumed ${ }^{240} \mathrm{Pu}$ spontaneous fission source and ${ }^{239} \mathrm{Pu}$ multiplying medium (red). Results are shown for increasing thicknesses of spherical polyethylene shell $\left(0.94 \mathrm{~g} \mathrm{~cm}^{-3}\right)$ around the ${ }^{252} \mathrm{Cf}$ (with increasing $\lambda^{-1}$, results are for 0 , 1, 2, 4, and $8 \mathrm{~cm}$ polyethylene, respectively). Also shown are estimated parameters for Source-1 based on assumed ${ }^{240} \mathrm{Pu}$ spontaneous fission 
source and ${ }^{239} \mathrm{Pu}$ multiplying medium (blue). These results are shown for increasing thicknesses of spherical polyethylene shell (0.94 $\mathrm{g}$ $\mathrm{cm}^{-3}$ ) around the plutonium metal (with increasing $\lambda^{-1}$, results are for $0,1,2,3$, and $4 \mathrm{~cm}$ polyethylene, respectively).

The second step of the authentication process is to re-analyze the Source-2 data using non-warhead item assumptions. Analysis of these results is trivial, as we know the source specification beforehand, but the results are illustrative and are shown in Figure 5.3 below. The new analysis yields parameters that are physical (maximum estimated efficiency $\varepsilon=$ 0.085 ) and correlated as expected (the decrease in efficiency with increasing moderation). These results are shown below in green, along with the previous results for comparison.
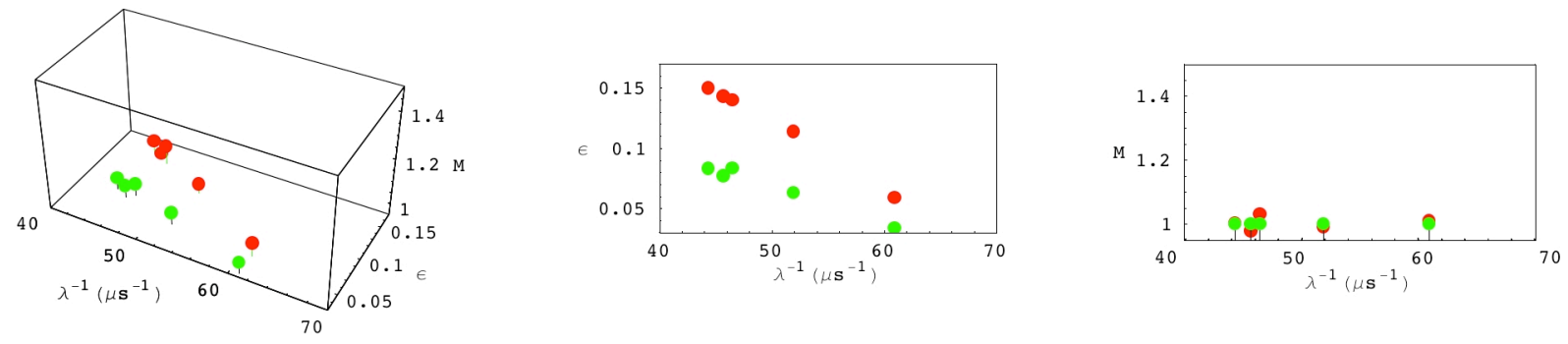

Figure 5.3. Estimated parameters $\lambda^{-1}, E$, and M for Source-2 $\left({ }^{252} C f\right.$ point source at the center of polyethylene sphere, for various sphere thicknesses), based on assumed ${ }^{252} \mathrm{Cf}$ spontaneous fission source (green) and ${ }^{240} \mathrm{Pu}$ spontaneous fission source within ${ }^{239} \mathrm{Pu}$ multiplying medium (red). Results are shown for increasing thicknesses of spherical polyethylene shell $\left(0.94 \mathrm{~g} \mathrm{~cm}^{-3}\right)$ around the ${ }^{252} \mathrm{Cf}$ (with increasing $\lambda^{-1}$, results are for $0,1,2,4$, and $8 \mathrm{~cm}$ polyethylene, respectively).

In this example, the Warhead Hypothesis would be rejected with extremely high confidence, based on the non-physical results from the warhead-assumption analysis, and the acceptable results of the non-warhead-assumption analysis.

For analysis with Source 3 , the moderated ${ }^{252} \mathrm{Cf}$-LEU source, we again conduct EFMT analysis of the LEU spoof data assuming it is a valid warhead-item. In this case, we assume the source is a ${ }^{240} \mathrm{Pu}$ spontaneous fission source within a ${ }^{239} \mathrm{Pu}$ multiplying medium. The results of this analysis are shown in Figures 5.5 and 5.6 and tabulated in Table 5.2 below, with Source 3 "spoof" results in orange, and Source 1 "warhead-item" results shown in blue for comparison. The parametric differences between the two sources are drastic, but it is important to note that in this case, the estimated multiplication $(\mathrm{M}=1.07)$ for the LEU spoof is both physical and reasonable for the assumed system, suggesting that $\mathrm{M}$, alone, is not a robust indicator of warhead authenticity. Rather, it is the deviation of the Efficiency-Moderation estimates from physical and acceptable ranges that indicates the item is not a valid warhead. In this important case, the EfficiencyModeration calculation (requiring a full EFMT analysis rather than basic NMC analysis) is the critical test of warhead authenticity. For the spoof source, estimated multiplication is far below the expected values (with non-physical results in some cases), and the Moderation-Efficiency results deviate up to a time-constant of roughly $60 \mu \mathrm{s}^{-1}$.
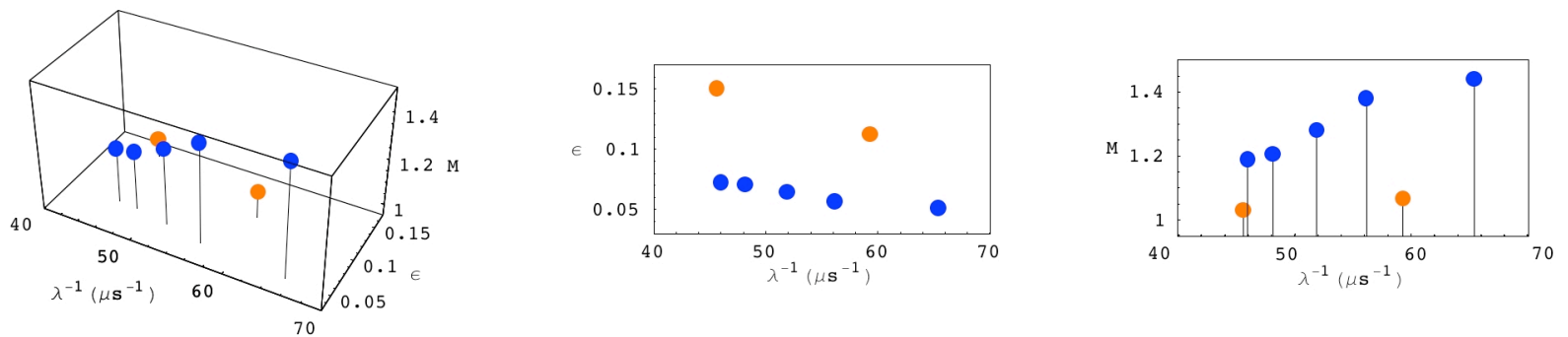

Figure 5.5. Estimated parameters $\lambda^{-1}, E$, and $M$ for Source $3\left({ }^{252} C f\right.$ point source at the center of polyethylene-reflected LEU sphere), based on assumed ${ }^{240} \mathrm{Pu}$ spontaneous fission source and ${ }^{239} \mathrm{Pu}$ multiplying medium (orange). Two cases are shown: bare and $4 \mathrm{~cm}$ polyethylene-reflected. Also shown are estimated parameters for Source-1 based on assumed ${ }^{240}$ Pu spontaneous fission source and ${ }^{239} \mathrm{Pu}$ multiplying medium (blue). These results are shown for increasing thicknesses of spherical polyethylene shell $\left(0.94 \mathrm{~g} \mathrm{~cm}{ }^{-3}\right)$ around the plutonium metal (with increasing $\lambda^{-1}$, results are for $0,1,2,3$, and $4 \mathrm{~cm}$ polyethylene, respectively). 


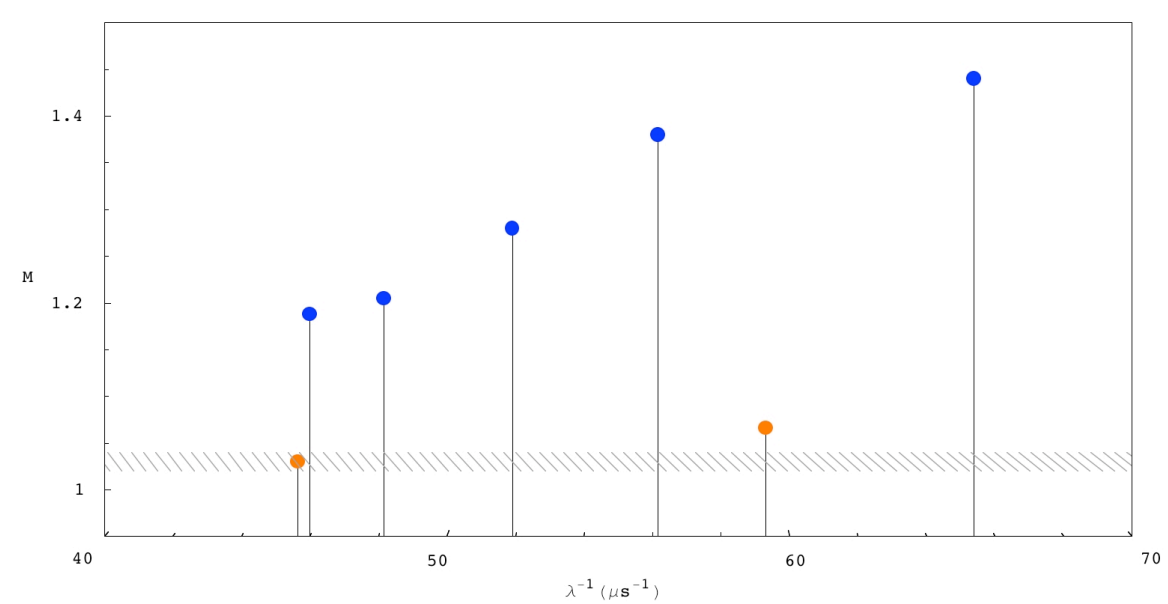

Figure 5.6. Estimated parameters $\lambda^{-1}$ and M for Source 3, based on assumed ${ }^{240}$ Pu spontaneous fission source and ${ }^{239} P u$ multiplying medium (orange). Also shown are estimated parameters for Source-1 based on assumed ${ }^{240} \mathrm{Pu}$ spontaneous fission source and ${ }^{239} \mathrm{Pu}$ multiplying medium (blue). The band $1.02<M<1.04$ represents a possible range of minimum acceptable values for $M$.

Results of the second step of the authentication process-EFMT analysis with spoof assumptions-are trivial and not shown, but fully support the rejection of the contained source as a warhead item.

\subsection{Estimating the Mass of the Measured Source}

Estimates of the source mass are based on assumed specific source rates for the materials and reactions of interest. The specific source rates used in the NMAC code are provided in Table 5.3, below.

Table 5.3. Specified specific neutron source intensities used in NMAC analysis.

\begin{tabular}{|cc|}
\hline Isotope & $\begin{array}{c}\text { NMAC Specific Spontaneous Fission } \\
\text { Neutron Intensity }\left(\mathrm{n} \mathrm{s}^{-1} \mathrm{~g}^{-1}\right)\end{array}$ \\
\hline${ }^{240} \mathrm{Pu}$ & 950 \\
${ }^{252} \mathrm{Cf}$ & $2.34 \times 10^{12}$ \\
\hline
\end{tabular}

Because the estimated mass is derived from the estimated source intensity using assumed specific intensities, it is most appropriate to consider the estimated source intensity as the primary measure of the ability to estimate mass. In our case, the Monte Carlo source intensity has been fixed at $22,500 \mathrm{n} \mathrm{s}^{-1}$. Analysis of the resulting time-tag data with NMAC yields estimates of the source strength. These results are presented in Table 5.4 below.

Table 5.4. Comparison of the estimated neutron source intensity and the intensity specified in the COG model, with the percentage deviation of the estimate from the defined value. The source intensity is a measure of the mass of ${ }^{240} \mathrm{Pu}$ or ${ }^{252} \mathrm{Cf}$, based on specific neutron intensity listed in Table 5.3.

\begin{tabular}{|llll|} 
& $\begin{array}{c}\text { Duration } \\
\text { (minutes) }\end{array}$ & $\begin{array}{c}\text { Estimated Source \% Difference } \\
\text { Rate }\left(\mathrm{n} \mathrm{s}^{-1}\right)\end{array}$ & $\begin{array}{l}\text { from 22,500 } \mathrm{n} \mathrm{s}^{-1} \\
\text { Warhead-items with Pu Assumptions }\end{array}$ \\
& & & \\
Warhead-item (Source 1) & & & 3.8 \\
Bare & 72.6 & 23360.5 & 2.8 \\
Polyethylene, $1 \mathrm{~cm}$ & 72.6 & 23123.0 & \\
\hline
\end{tabular}




\begin{tabular}{|c|c|c|c|}
\hline Polyethylene, $2 \mathrm{~cm}$ & 145.0 & 23484.0 & 4.4 \\
\hline Polyethylene, $3 \mathrm{~cm}$ & 144.7 & 24225.0 & 7.7 \\
\hline Polyethylene, $4 \mathrm{~cm}$ & 232.6 & 23997.0 & 6.7 \\
\hline \multicolumn{4}{|c|}{ Spoofs with Proper Assumptions } \\
\hline \multicolumn{4}{|l|}{${ }^{252} \mathrm{Cf}$ (Source 2) } \\
\hline Bare & 74.5 & 23212.8 & 3.2 \\
\hline Polyethylene, $1 \mathrm{~cm}$ & 7.6 & 21925.8 & 2.6 \\
\hline Polyethylene, $2 \mathrm{~cm}$ & 7.5 & 22581.0 & 0.4 \\
\hline Polyethylene, $4 \mathrm{~cm}$ & 74.3 & 22698.0 & 0.9 \\
\hline Polyethylene, $8 \mathrm{~cm}$ & 74.2 & 23306.4 & 3.6 \\
\hline \multicolumn{4}{|c|}{ LEU Spoof (Source 7) } \\
\hline Bare & 74.3 & 22698.0 & 0.9 \\
\hline Polyethylene, $4 \mathrm{~cm}$ & 233.8 & 22698.0 & 0.9 \\
\hline \multicolumn{4}{|c|}{ Spoofs with Pu Assumption } \\
\hline \multicolumn{4}{|l|}{${ }^{252} \mathrm{Cf}$ (Source 2) } \\
\hline Bare & 72.6 & 12578.0 & 44.1 \\
\hline Polyethylene, $1 \mathrm{~cm}$ & 72.6 & 12825.0 & 43.0 \\
\hline Polyethylene, $2 \mathrm{~cm}$ & 145.0 & 12312.0 & 45.3 \\
\hline Polyethylene, $4 \mathrm{~cm}$ & 144.7 & 12616.0 & 43.9 \\
\hline Polyethylene, $8 \mathrm{~cm}$ & 232.6 & 13262.0 & 41.1 \\
\hline \multicolumn{4}{|c|}{ LEU Spoof (Source 7) } \\
\hline Bare & 74.3 & 12701.5 & 43.5 \\
\hline Polyethylene, $4 \mathrm{~cm}$ & 233.8 & 12540.0 & 44.3 \\
\hline
\end{tabular}

The results show that the EFMT analysis yields source-mass estimates in good agreement with the model definitions - 3\% average deviation with the largest difference less than $8 \%$. These are statistical errors as calculated in the Monte Carlo simulation. The uncertainties in the estimates of source strength tend to be on the order of $1 \%$ or less for the models considered.

Of course, these estimates depend on making proper assumptions for the source isotope and multiplying medium. However, it is reasonable to expect that with a limited set of possible sources $\left({ }^{240} \mathrm{Pu},{ }^{252} \mathrm{Cf},(\alpha, \mathrm{n})\right)$ and multiplying materials $\left({ }^{239} \mathrm{Pu},{ }^{235} \mathrm{U}\right)$ the assumptions will be correct, and that any improper assumptions would be revealed by the results of the Efficiency-Moderation-Multiplication analysis. Complications will arise, however, for composite sources and/or combinations of different multiplying isotopes. In these cases, more detailed gamma ray spectroscopy might be required to determine source isotopics. 


\subsection{Indication of the Presence of High-Explosive}

As discussed previously, the system time constant is a measure of the moderation of the source-container-detector system. The time-constant can be estimated directly from the EFMT "sliding time-scale" data—an important advantage of EFMT over NMC.

We expect some elevation in the time constant due to cushioning/stablilizing material in the containers, such as celotex. The presence and amount of this material would be fixed as an agreed term of a monitoring regime. Any additional increase in the time-constant would indicate additional moderating material between the source and detector. This material would not necessarily be high explosive, but could be other low atomic mass material such as polyethylene or beryllium. Results from Monte Carlo modeling suggest that the EFMT approach can detect increased moderation timeconstants consistent with the presence of polyethylene or high-explosive. Moreover, our results suggest that timeconstants above roughly $50 \mathrm{\mu s}^{-1}$ (for the modeled system) would indicate beryllium or graphite shielding thicknesses greater than 4-6 cm, as shown in Figure 5.7 below.

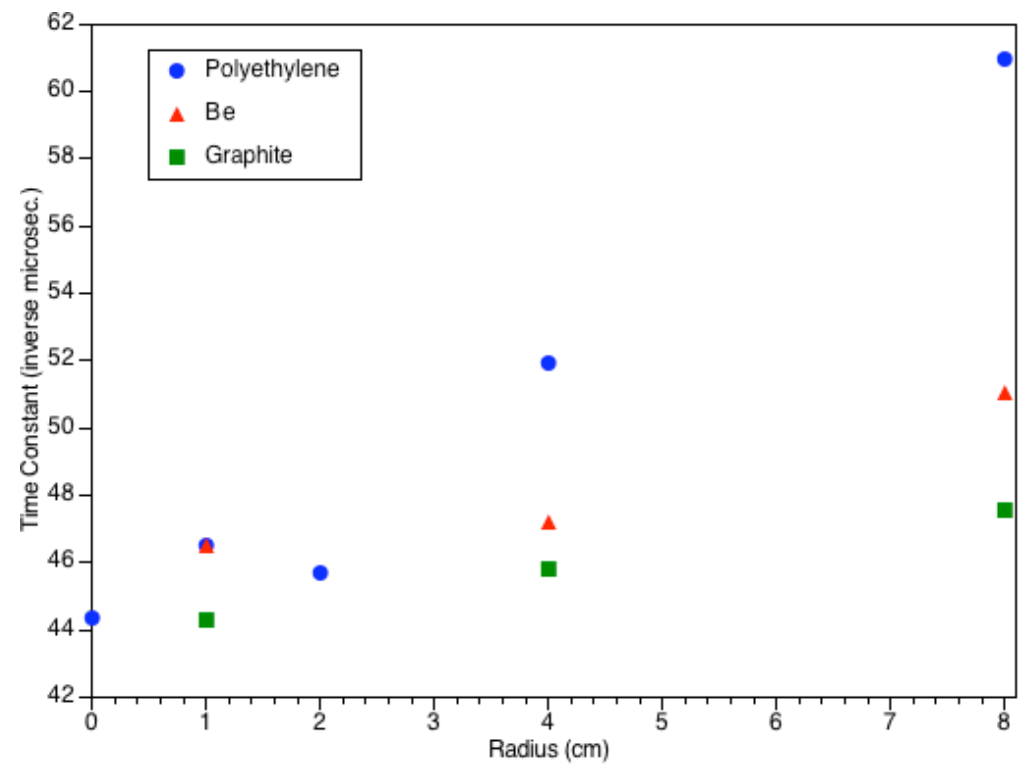

Figure 5.7. Monte Carlo results for the effect of varying shielding thickness on time-constant $\lambda^{-1}$, for $a^{252} C f$ point source in spherical shielding. Results are shown for polyethylene $\left(\rho=0.94 \mathrm{~g} / \mathrm{cm}^{3}\right)$, beryllium $\left(\rho=1.85 \mathrm{~g} / \mathrm{cm}^{3}\right)$, and graphite $\left(\rho=2.25 \mathrm{~g} / \mathrm{cm}^{3}\right)$ shielding.

\section{CONCEPTUAL REQUIREMENTS FOR A WARHEAD AUTHENTICATION MEASUREMENT INSTRUMENT WITH INFORMATION BARRIER BASED ON THE EXTENDED FEYNMAN TECHNIQUE;}

The forgoing analysis and concept of operation allows us to define a set of high-level requirements covering the key functions of a measurement device suitable for safeguards monitoring, transparency, or arms control based on the Extended Feynman Moment Technique. Before specifying a fieldable instrument, however, these requirements should undergo a review process that includes participants from the radiation detection, onsite inspection, and data security communities. Meant to establish topical areas, these broad requirements should be decomposed into testable statements before using them to write instrument specifications. Nonetheless, the following should suffice as guidelines for the construction of a prototype demonstration system.

1. The system shall determine the neutron multiplication attributes of material present in a measured item. (This information derives from count distribution analysis, and can be readily gathered with commercial equipment containing fast electronics.) 
2. The system shall determine the mass of fissile material present in a measured item. (The mass of the spontaneously fissioning nuclides derives from the measured reaction rate. The overall mass of fissile material may be inferred from knowledge of the isotopic ratios or from independent measurement of them.)

3. The system shall determine the moderating properties of a measured item. (This attribute derives from the variation in the calculated second moments as a function of time gate width.)

4. The system shall prevent disclosure of any information beyond agreed attributes or template comparison. In particular, the system shall purge all measurement data upon detection of error or attempt to access said data.

5. The system shall operate autonomously.

6. The system shall retain measurement data only long enough to extract the measurement attributes or templates.

7. The system shall provide for a process of self-authentication, wherein the correct functioning of the system can be verified.

8. The system shall perform all measurements in a single mode. In particular, the measurement and analysis portions of the system shall not be capable of distinguishing item assay from self-authentication functions.

9. The system shall follow a prescribed security policy with respect to operator controls and indications.

10. The system shall be designed to provide reliable indications within defined margins of measurement parameters (distance, source strength). 


\section{APPENDIX A. EVALUATION AND VALIDATION OF THE MONTE CARLO - NMAC METHOD}

Overall, we have tested our approach extensively, taking care to ensure that the simulated results are physical, internally consistent, and in good agreement with theory and experiment. Results from the correlation method are sensitive functions of certain physical parameters, and care must be taken to ensure that the relevant physical processes are represented properly and with accuracy.

Moreover, out method must be evaluated further, and the results verified by both 1) simulating simple test cases, the results of which can be compared to theoretical results, and 2) simulating complex experimental configurations and comparing the results to qualitative experimental results.

\section{A.1. Physical Self-Consistency}

The first test of the validity of the Monte Carlo model is to confirm that the simulated results are self-consistent with the values entered as parameters into the code (to within stochastic uncertainty). In this case, we test that the ratio of parameters for spontaneous fission source rate and spontaneous fission multiplicity distribution is in agreement with the ratio of results for total count rate and correlated doubles count rate. That is, from EFMT theory we test the equality:

$$
\frac{S_{s f}}{D_{2 s}} \stackrel{?}{=} \frac{R_{1}}{R_{2 F}}
$$

where

$$
\begin{aligned}
& S_{s f}=\text { fission rate } \times \bar{v} \\
& D_{2 s}=\frac{\Gamma_{2}}{2} \bar{v} \\
& R_{1}=\text { total count rate } \\
& R_{2 F}=\text { doubles count rate } \\
& \Gamma_{2}=\left(\left\langle v^{2}\right\rangle_{A v}-\bar{v}\right) / \bar{v}^{2}
\end{aligned}
$$

A COG model similar to that described in the main body of this report, but with two rather than four pods and a bare point-source of ${ }^{252} \mathrm{Cf}$, was used evaluate self-consistency. The values of the two parameters and two derived quantities are provided in Table A.1, below.

Table A1. Summary of results derived from four-pod model

\begin{tabular}{|lll|}
\hline Parameter or Variable & Value & Comments \\
\hline $\bar{v}$ & 3.86 & ${ }^{252} \mathrm{Cf}$ \\
$\mathrm{S}_{\mathrm{sf}}$ & $2.1391 \times 10^{5} \mathrm{~s}^{-1}$ & From $\bar{v}$ and fission rate $=5.5416 \mathrm{E}^{-1} \mathrm{~s}^{-1}$ \\
$\Gamma_{2}$ & 0.825 & $\begin{array}{l}\text { Ref. Terrell, }{ }^{252} \mathrm{Cf} \text {, based on Gaussian } \\
\text { distribution with } \sigma=1.08^{7} .\end{array}$ \\
$\mathrm{D}_{2 \mathrm{~s}}$ & 1.59225 & $\frac{\Gamma_{2}}{2} \bar{v}$ \\
$\mathrm{R}_{1}$ & $2.5383 \mathrm{E}^{2} \mathrm{~s}^{-1}$ & NMAC result \\
$\mathrm{R}_{2 \mathrm{~F}}$ & 0.188 & NMAC result
\end{tabular}


The difference between the simulated ratio and the parametric ratio, then, is:

$$
\frac{\left|\frac{S_{s f}}{D_{2 s}}-\frac{R_{1}}{R_{2 F}}\right|}{\frac{S_{s f}}{D_{2 s}}}=4.8 \times 10^{-3}
$$

which is equivalent to an estimated deviation of the model results from defined parameters of less than 0.5 percent. This difference is readily attributed to Monte Carlo uncertainty, and strongly supports the assertion that the COG code is adequately reproducing the physical internal-consistency of spontaneous fission, neutron transport, and detection phenomena as they relate to neutron correlations.

\section{A.2. Qualitative Effect of Moderator Absorption on the Feynman Moment Curve}

Experiments by Kerr and Snyderman ${ }^{\dagger}$ show that the Feynman curves (particularly that of the second moment, or $\mathrm{Y}_{2 \mathrm{~F}}$ ) exhibit non-point source diffusion behavior with increasing neutron absorption in the moderating material. The effect is a more rapid rise in the observed $\mathrm{Y}_{2 \mathrm{~F}}$ curve than is explained by a fit to the point diffusion model. This can be explained by an increase in the thermal neutron capture by hydrogen in the increased mass of polyethylene. This phenomenon is akin to "absorption heating" observed in the thermal neutron flux in nuclear reactors; as neutrons are slowed down, they are more likely to be absorbed. The net effect is an increase in the average neutron energy relative to predictions based on flux in non-absorbing media. In terms of the neutron correlation, the result is an increase in the average velocity of detected neutrons by preferential elimination (absorption) of very slow neutrons. This, in turn, reduces the fraction of counts from neutrons that exhibit long-period time correlations, and leads to a faster rise in the $Y_{2 F}$ curve than predicted by point diffusion.

This phenomenon has been reproduced in the COG simulations and is evident in Figure A.1, which shows the COG- and NMAC-generated $\mathrm{Y}_{2 \mathrm{~F}}$ curves for standard 4-pod detection configuration with a ${ }^{252} \mathrm{Cf}$ point source placed inside a sphere of polyethylene of radius $8 \mathrm{~cm}$. The simulated results show a faster rise profile than the point diffusion fit to the data. This difference is accentuated by limiting the fit of the diffusion prediction to time-gates greater than $200 \mu$ s. Based on the experimental results, the typical deviation from the point-diffusion model is under-prediction of the second moment up to roughly $200 \mu \mathrm{s}$, followed by a region of over-prediction from $200 \mu \mathrm{s}$ to $400 \mu \mathrm{s}$, with a final region of underprediction from $400 \mu$ s to $512 \mu \mathrm{s}$. This has been reproduced using the COG model.

\footnotetext{
${ }^{\dagger}$ Personal correspondence.
} 


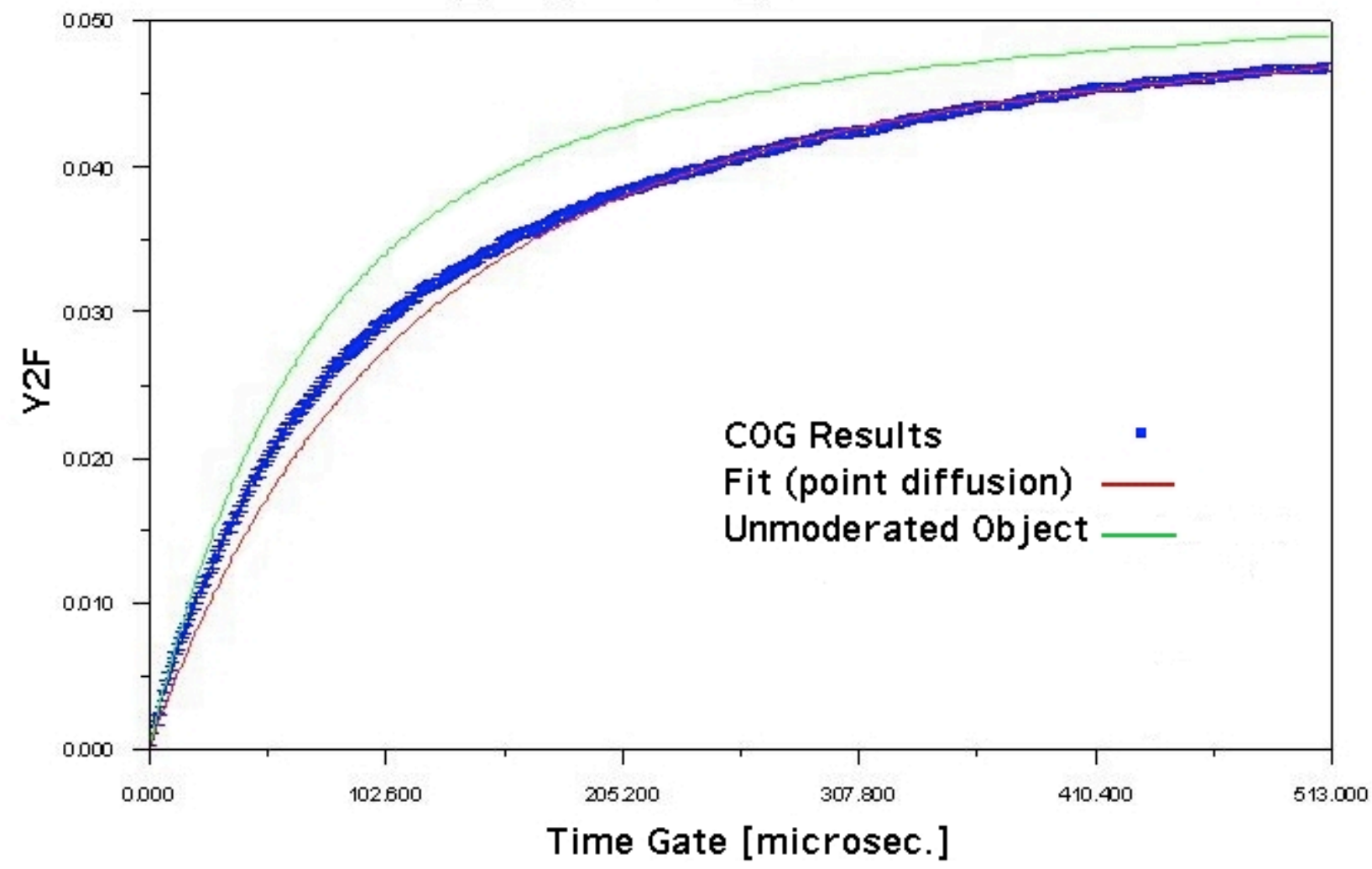

Figure A.1. Monte Carlo results for the effect of varying shielding thickness on time-constant $\lambda^{-1}$, for a ${ }^{252}$ Cf point source in spherical shielding. Results are shown for polyethylene $(\rho=0.94)$, beryllium $(\rho=1.85)$, and graphite $(\rho=2.25)$ shielding.

\section{ACKNOWLEDGMENTS}

The authors wish to thank Drs. Neal Snyderman and Manoj Prasad for their expert advice in our calculation of the Feynman moments, as well as access to their software tools, NMAC and BIGFIT. Without their guidance, this work would not have been possible. This work was completed by Lawrence Livermore National Laboratory for the U.S. Department of Energy under contract W-7405-ENG-48.

\section{REFERENCES}

1. Prasad, M.K. and Snyderman, N.J., Statistical Theory of Fission Chains and Generalized Poisson Neutron Counting Distributions, Lawrence Livermore National Laboratory, UCRL-ID-148010, April 16, 2002.

2. Ensslin, N., Harker, W.C., Krick, M.S., Langer, D.G., Pickrell, M.M., Stewart, J.E., Application Guide to Neutron Multiplicity Counting, Los Alamos National Laboratory, LA-13422-M, UC-700, November, 1998.

3. Buck, R., Lent, E., Wilcox, T., COG-A Multiparticle Monte Carlo Transport Code, User's Manual, 5th ed., LLNL (2002).

4. NEA Nuclear Science Committee, International Handbook of Evaluated Criticality Safety Benchmark Experiments, Nuclear Energy Agency, Organization for Economic Cooperation and Development, NEA/NSC/DOC(95)03, September 2002 Edition. 
5. James S. Baker and Andrei V. Belooussov, Criticality Analysis for the United States Safety Evaluation of the Russian Fissile Material Storage Facility, Los Alamos National Laboratory, LA-CP-00-410,October 31. 2000.

6. Cifarelli, D.M. and Hage, W., "Three-parameter analysis of neutron signal correlation measurements", Nuclear Instruments and Methods, A251 (1986) 550.

7. Terrell, J., "Distributions of Fission Neutron Numbers", Physical Review, Vol. 108, No. 3, Nov. 1, 1957. See p. 786, Table I. 
Unuversily of Califormia

Lawrence Livermere Natienal Laboratery

Technical InformationDepartment

Livermore, CA 94551

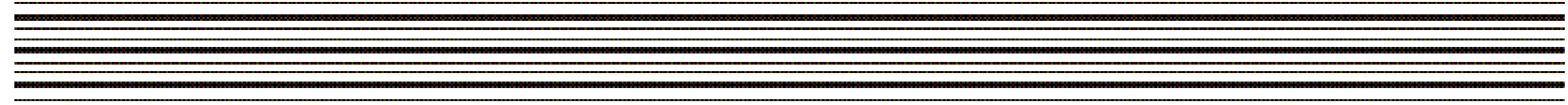

